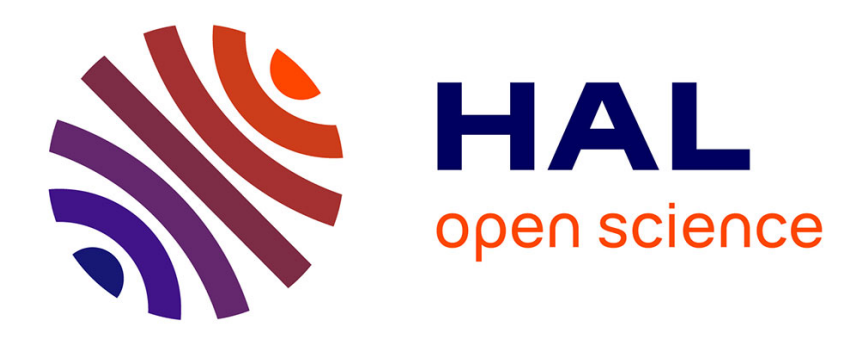

\title{
Structures of Opposition and Comparisons: Boolean and Gradual Cases
}

Didier Dubois, Henri Prade, Agnès Rico

\section{To cite this version:}

Didier Dubois, Henri Prade, Agnès Rico. Structures of Opposition and Comparisons: Boolean and Gradual Cases. Logica Universalis, 2020, 14 (1), pp.115-149. 10.1007/s11787-020-00241-6 . hal03121904

\section{HAL Id: hal-03121904 \\ https://hal.science/hal-03121904}

Submitted on 26 Jan 2021

HAL is a multi-disciplinary open access archive for the deposit and dissemination of scientific research documents, whether they are published or not. The documents may come from teaching and research institutions in France or abroad, or from public or private research centers.
L'archive ouverte pluridisciplinaire HAL, est destinée au dépôt et à la diffusion de documents scientifiques de niveau recherche, publiés ou non, émanant des établissements d'enseignement et de recherche français ou étrangers, des laboratoires publics ou privés. 


\title{
Structures of opposition and comparisons. Boolean and gradual cases
}

\author{
Didier Dubois, Henri Prade and Agnès Rico
}

\begin{abstract}
This paper first investigates logical characterizations of different structures of opposition that extend the square of opposition in a way or in another. Blanché's hexagon of opposition is based on three disjoint sets. There are at least two meaningful cubes of opposition, proposed respectively by two of the authors and by Moretti, and pioneered by philosophers such as J. N. Keynes, W. E. Johnson, for the former, and H. Reichenbach for the latter. These cubes exhibit four and six squares of opposition respectively. We clarify the differences between these two cubes, and discuss their gradual extensions, as well as the one of the hexagon when vertices are no longer two-valued. The second part of the paper is dedicated to the use of these structures of opposition (hexagon, cubes) for discussing the comparison of two items. Comparing two items (objects, images) usually involves a set of relevant attributes whose values are compared, and may be expressed in terms of different modalities such as identity, similarity, difference, opposition, analogy. Recently, J.-Y. Béziau has proposed an "analogical hexagon" that organizes the relations linking these modalities. Elementary comparisons may be a matter of degree, attributes may not have the same importance. The paper studies in which ways the structure of the hexagon may be preserved in such gradual extensions. As another illustration of the graded hexagon, we start with the hexagon of equality and inequality due to R. Blanché and extend it with fuzzy equality and fuzzy inequality. Besides, the cube induced by a tetra-partition can account for the comparison of two items in terms of preference, reversed preference, indifference and non-comparability even if these notions are a matter of degree. The other cube, which organizes the relations between the different weighted qualitative aggregation modes, is more relevant for the attribute-based comparison of items in terms of similarity.
\end{abstract}

Mathematics Subject Classification (2010). Primary 68T30; Secondary 03A05, 03B05, 68T37.

The authors acknowledge a partial support of ANR-11-LABX-0040-CIMI (Centre International de Mathématiques et d'Informatique) within the program ANR-11-IDEX-0002-02, project ISIPA. 
Keywords. Square of opposition, hexagon of opposition, cube of opposition, partition, difference, similarity, analogy, ordered weighted min, comparison, preferences.

\section{Introduction}

The square of opposition [45] is a logical structure introduced in Ancient Greek logic, starting with Aristotle, in relation with the study of syllogisms [45]. It has greatly influenced medieval logic, as for instance, in the treatises by William of Sherwood (c.1200-c.1272) [54] or by Charles de Bovelles (1479-1566?) [17]. The square is still present in textbooks of the beginning of the XVII ${ }^{\text {th }}$ century (e.g., [33]), but, one century and half later, in the Encyclopédie of Diderot and d'Alembert [2], only the logical expressions associated to the four corners of the square remain, with their traditional names $\mathrm{A}, \mathrm{I}, \mathrm{E}, \mathrm{O}$, but the square is no longer pictured. It partially fell into oblivion in XIX $^{\text {th }}$ century with the advent of modern logic, although philosophers like Keynes [39] and Johnson [38] still discussed syllogisms on the basis of the square and introduced some extensions of it in the form of an octagon.

The square was studied again in philosophical logic in the 1960's due to Robert Blanché's discovery of the hexagon of opposition [15], independently of some forerunners $[37,53]$. The hexagon includes three squares of opposition, and is a scheme that agrees with the structural organization of many examples of related concepts. This hexagon is based on three disjoint sets; see for instance [25].

Nevertheless, the interest in the square and its extensions has been revived at the beginning of the new century $[6,10,14,11,12,13]$. A cube of opposition relying on a quadri-partition and including six squares of opposition was proposed by Moretti $[42,43]$ a decade and half ago. In fact, it had appeared once in 1952, in a thorough discussion of syllogisms by Reichenbach [49], but remained largely ignored since then. Another cube of opposition, originated in the works of Keynes [39] and Johnson [38] has been rediscovered some years ago [25] when trying to relate possibility theory $[58,26]$ with the square of opposition. In this cube, two opposite facets are squares of opposition (and there two other non-planar squares inside the cube), while the remaining facets exhibit other noticeable structures. A careful analysis of the differences between these two cubes is yet to be carried out. As it turns out, Moretti's cube expresses more constraints than the other one. Moreover, we investigate the link between these structures of opposition and their associated partitions.

The square, the hexagon, and the two cubes mentioned above are binaryvalued structures. They become gradual when the formal expressions associated to their vertices are a matter of degree. With the exception of Moretti's cube, some gradual extensions of these structures have been already proposed 
and applied to several quantitative settings including possibility theory, belief functions, weighted multiple criteria aggregations, fuzzy rough sets, and fuzzy relations $[28,18,19]$.

In the second part of this paper, we apply the graded hexagon and the two graded cubes to concepts pertaining to the comparison of two objects, which may be a matter of degree. In order to compare two objects, two images, etc. (we shall say items, more generally), it is natural to refer to their descriptions. Descriptions are then understood as plain lists of supposedly relevant attribute values. The two items are assumed to be described by the same set of attributes and the values of these attributes are supposed to be known. One is then naturally led to state that two items are identical if their respective values for each relevant attribute coincide. Béziau [9] recently pointed out that the notion of identity, along with five other modalities pertaining to comparison (opposition, similarity, difference, analogy, non-analogy), form a hexagon of opposition. This hexagon-driven approach yields an organized overview of a family of logically related operators. The study of the compatibility between fuzzy extensions of comparison operations and the logical hexagon is thus worth investigating. Besides, the gradual extension of Johnson-Keynes cube, which fits with weighted aggregations, is also relevant for the comparison of items when features have unequal importance. In contrast, Moretti's cube, based on quadri-partition, applies to binary preferences [50] since when comparing two items $a$ and $b$, four attitudes are possible: i) one may prefer $a$ to $b$, ii) one may prefer $b$ to $a$, iii) one may be indifferent between $a$ and $b$, and iv) one may find $a$ and $b$ not comparable. However the four relations may be a matter of degree $[35,56]$.

This paper is divided in two main parts. Section 2 provides a unified view of the hexagon and cubes associated with the square of opposition, including their gradual extensions. In particular the gradual extension of Moretti's cube is presented. Section 3 is devoted to the application of structures of opposition to the comparisons of items. It first presents the fuzzy extension of Blanché's hexagon for inequality and equality operators, and then provide two examples of quantitative hexagons for similarity indices based on cardinalities. The section then focuses on logical expressions agreeing with Béziau's analogy hexagon, and then on various possible fuzzy extensions. This provides a structure relating gradual indices of opposition, similarity, difference, analogy, and non-analogy. Such gradual extensions take into account approximate equality, and attribute importance. A preliminary version of that latter part of the section appeared in [32]. We finally consider the two cube structures and indicate their relevance for the comparison of items.

\section{Structures of opposition and partitions}

This section reviews the structures of opposition (square, hexagon, cubes). It provides their logical characterization and their gradual extensions. We begin by a presentation of the square of opposition, before considering the hexagon 
of opposition, and then Moretti's cube, and finally a structure proposed by Johnson and Keynes and retrieved by Dubois and Prade.

\subsection{The square of opposition and its gradual extension}

The traditional square of opposition [45] is built with universally and existentially quantified statements in the following way. Consider a statement (A) of the form "all $P$ 's are $Q$ 's", which is negated by the statement $(\mathbf{O})$ "at least one $P$ is not a $Q$ ", together with the statement $(\mathbf{E})$ "no $P$ is a $Q$ ", which is clearly in even stronger opposition (than $\mathbf{O}$ ) to the first statement (A). These three statements, together with the negation of the last statement, namely $(\mathbf{I})$ "at least one $P$ is a $Q$ " can be displayed on a square whose vertices are traditionally denoted by the letters A, I (AffIrmative half: from Latin "AffIrmo") and E, O (nEgative half: from Latin "nEgO"), as pictured in Figure 1 (where $\bar{Q}$ stands for "not $Q$ ").

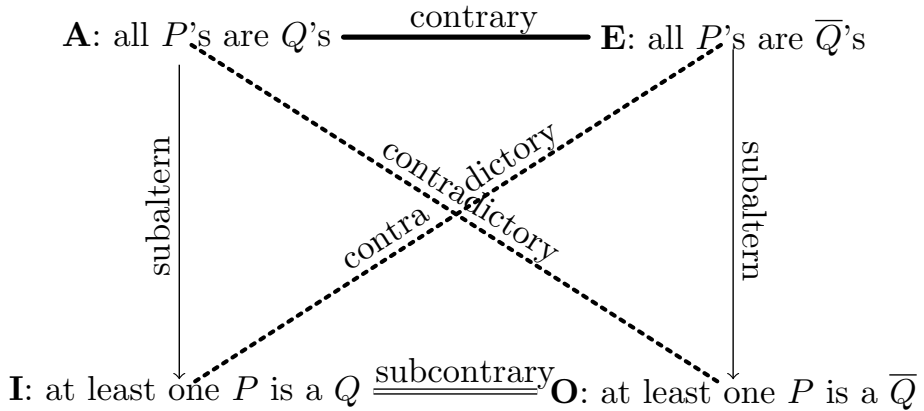

Figure 1. Square of opposition

As can be checked, noticeable relations hold in the square:

(i) $\mathbf{A}$ and $\mathbf{O}$ (resp. $\mathbf{E}$ and $\mathbf{I}$ ) are the negation of each other;

(ii) A entails $\mathbf{I}$, and $\mathbf{E}$ entails $\mathbf{O}$ (it is assumed that there is at least one $P$ for avoiding existential import problems);

(iii) $\mathbf{A}$ and $\mathbf{E}$ cannot be true together;

(iv) $\mathbf{I}$ and $\mathbf{O}$ cannot be false together.

Note that there is no constraint on the falsity of $\mathbf{A}$ and $\mathbf{E}$ in (iii) (either one of them, or both, may be false), nor on the truth of $\mathbf{I}$ and $\mathbf{O}$ in (iv) (either one of them, or both, may be true). So we can formally state, using the standard propositional connectives $\neg, \vee, \wedge, \rightarrow$, 三:

Definition 1. In a square of opposition AEOI, the following holds:

(a) The diagonal link between $\mathbf{A}$ and $\mathbf{O}$, symmetrically relate contradictories. It expresses that $\mathbf{A}$ and $\mathbf{O}$ are the negation of each other namely, $\mathbf{A} \equiv \neg \mathbf{O}$ holds. Similarly, $\mathbf{E} \equiv \neg \mathbf{I}$.

(b) The vertical arrows represent entailment relations corresponding to implications $\mathbf{A} \rightarrow \mathbf{I}=\neg \mathbf{A} \vee \mathbf{I}$ and $\mathbf{E} \rightarrow \mathbf{O}=\neg \mathbf{E} \vee \mathbf{O}$. 
(c) The link between $\mathbf{A}$ and $\mathbf{E}$ which represents the symmetrical relation of contrariety, corresponds to mutual exclusion, namely $\neg \mathbf{A} \vee \neg \mathbf{E}$ should hold.

(d) The link between $\mathbf{I}$ and $\mathbf{O}$ which represents the symmetrical relation of subcontrariety, is a disjunction, namely $\mathbf{I} \vee \mathbf{O}$ holds.

This leaves us with three options for defining a formal square of opposition with independent conditions [19]: i) either we can regard (a) and (b), or ii) (a) and (c), or iii) (a) and (d), as the basic requirements. Actually, we can express the content of a square of opposition in propositional logic using two propositional atoms (say $\mathbf{A}$ and $\mathbf{E}$ ) and a knowledge base containing only the axiom $\neg \mathbf{A} \vee \neg \mathbf{E}$, expressing their mutual exclusion (and then defining $\mathbf{I} \equiv \neg \mathbf{E}$ and $\mathbf{O} \equiv \neg \mathbf{A})$.

\subsection{The graded square of opposition}

We now recall the construction of the graded square of opposition [27, 19, 31]. We attach four variables $\alpha, \epsilon, o, \iota$, valued on a bounded totally ordered set $L$, to vertices $\mathbf{A}, \mathbf{E}, \mathbf{O}, \mathbf{I}$ respectively. $L$ has top 1 and bottom 0 and is assumed to be equipped with an involutive negation $n: L \rightarrow L$. The involutive nature of the negation is essential in the definition of the square of opposition because of the expected symmetry between contradictories.

In order to keep the properties of the square in the gradual case, the negation, the implication, the conjunction and the disjunction must be linked. More precisely, given that $\alpha$ and $o$ are the negation each other as well as $\epsilon$ and $\iota$, the properties $i(\alpha, \iota)=1$ and $i(\epsilon, o)=1, c(\alpha, \epsilon)=0$ and $d(\iota, o)=1$ must be equivalent. This is the case, as shown in [31], if we consider the following triplet $(i, c, d)$ of binary operations related via the negation:

conjunction. $c: L \times L \rightarrow L$ coincides with a Boolean conjunction on $\{0,1\}$;

it is increasing in both places.

implication. $i: L \times L \rightarrow L$ coincides with a Boolean implication on $\{0,1\}$;

it is is decreasing in the first place and increasing in the second place.

semi-duality. $i$ and $c$ are mutually definable by:

$$
i(x, y)=n(c(x, n(y))) \Longleftrightarrow c(x, y)=n(i(x, n(y)))
$$

disjunction. $d: L \times L \rightarrow L$ is associated with $c$ by De-Morgan duality:

$$
d(x, y)=n(c(n(x), n(y))) .
$$

We are now in a position to state the definition of a graded square of opposition:

Definition 2. Given an involutive negation $n: L \rightarrow L$, a triplet $(i, c, d)$ of implication, conjunction and disjunction defined as above, a graded square of opposition $\alpha \epsilon O \iota$ respects the following constraints:

(a) $\alpha$ and $o$ (resp. $\epsilon$ and $\iota)$ are each other's negation:

$$
o=n(\alpha) \text { and } \iota=n(\epsilon) \text {. }
$$


(b) A subaltern relationship between $\alpha$ and $\iota$ (resp. $\epsilon$ and $o$ ):

$$
i(\alpha, \iota)=1 \text { and } i(\epsilon, o)=1 \text {. }
$$

(c) There is mutual exclusion between $\alpha$ and $\epsilon$, i.e., they cannot be simultaneously equal to 1 :

$$
c(\alpha, \epsilon)=c(\epsilon, \alpha)=0 .
$$

(d) $\iota$ and $o$ must cover all situations:

$$
d(\iota, o)=d(o, \iota)=1 \text {. }
$$

Note that condition (c) does not prevent the situation where $\alpha=\epsilon=0$; and likewise, condition (d) does not prevent the situation where $\iota=o=1$.

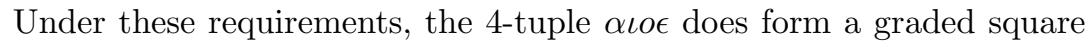
of opposition. Note that we can also observe a graceful degradation of the square properties when $i(\alpha, \iota)<1$, namely, it can be checked that the smaller $i(\alpha, \iota)$ (i.e., $\alpha$ increases while $\iota$ decreases), the greater $c(\alpha, \epsilon)=n(i(\alpha, n(\iota))$.

There are two ways of generating squares of from a four-tuple of negation, implication, conjunction, and disjunction. We can start with a conjunction and an involutive negation, and derive the implication by semi-duality and the disjunction by De Morgan duality, or start with an implication and an involutive negation, and derive the conjunction by semi-duality and the disjunction by De Morgan duality.

If we start with a conjunction $c$, the following result holds:

Proposition 1. If the conjunction $c$ has no zero divisors, then the obtained square of opposition is partially Boolean.

Proof A conjunction $c$ has no zero divisors means that $c(x, y)=0$ if and only if $x=0$ or $y=0$. So the condition $(c)$ pertaining to contraries, i.e., $c(\alpha, \epsilon)=c(\epsilon, \alpha)=0$ which implies that $\alpha=0$ or $\epsilon=0$. If $\alpha=0$ then $o=1$ too.

As a consequence if we choose for $c$ a triangular norm [40], that is, a continuous two-place conjunction on $[0,1]$ that is commutative, associative, and such that $c(x, 0)=0, c(x, 1)=x$, then Proposition 1 applies if $c=\min$ or $c$ is a strict triangular norm like the product.

Example 1. [27] Take min for conjunction, and the negation $1-(\cdot)$ on the scale $[0,1]$, one obtains the Kleene system of operations. It leads to a graded square of opposition with conditions:

(a) $\alpha=1-o, \epsilon=1-\iota$

(b) $\max (1-\alpha, \iota)=1, \max (1-\epsilon, o)=1$ (using Kleene-Dienes implication).

(c) $\min (\alpha, \epsilon)=0$

(d) $\max (\iota, o)=1$

As expected, only half of the square is graded because either one must have that $\alpha=0$ (hence $o=1$ ) or $\epsilon=0$ (hence $\iota=1$ ).

In contrast we have the following result: 
Proposition 2. If the conjunction $c$ is such that $c(x, y)=0$ if and only if $x \leq n(y)$, then the triplet $(c, i, d)$ obtained by semi-duality and De Morgan duality yields a genuine graded square of opposition.

Proof The required conditions for a graded square of opposition take the form:

(c) $c(\alpha, \epsilon)=0$ which is equivalent to $\alpha \leq n(\epsilon)$

(b) as $\alpha=n(o), \epsilon=n(\iota)$, we get $i(\epsilon, o)=n(c(\epsilon, n(o))=n(c(\epsilon, \alpha))=1$ since $\epsilon \leq n(\alpha)$. And likewise we get $i(\alpha, \iota)=1$.

(d) $d(\iota, o)=1$ is obvious.

An important class of examples illustrating the last result uses a residuated implication $i=\rightarrow_{*}$ obtained from a symmetric conjunction $*$ by as $x \rightarrow_{*} y=\inf \{z: x * z \leq y\}$ (for instance see [34]). It is such that $i(x, y)=1$ if and only if $x \leq y$, and so for the semi-dual conjunction, it holds that $c(x, y)=n\left(x \rightarrow_{*} n(y)\right)$ with $c(x, y)=0$ if and only if $x \leq n(y)$.

Example 2. [31] Take min for $*$ and the negation $n=1-(\cdot)$ on $[0,1]$. Take $\rightarrow_{*}=\rightarrow_{G}$, the Gödel implication, such that $x \rightarrow_{G} y=\left\{\begin{array}{l}y, \text { if } x>y \\ 1 \text { otherwise }\end{array}\right.$. Let $i=\rightarrow_{G}, c(x, y)=x \otimes_{G} y=1-\left(x \rightarrow_{G}(1-y)\right)=y$ if $x>1-y$ and 0 otherwise, and $d(x, y)=x \oplus_{G} y=1-(1-x) \otimes_{G}(1-y)$, then one obtains the Gödel square of opposition, provided that $\alpha+\epsilon \leq 1$ :

(a) $\alpha=1-o, \epsilon=1-\iota$

(b) $\alpha \rightarrow_{G} \iota=1, \epsilon \rightarrow_{G} o=1$.

(c) $\alpha \otimes_{G} \epsilon=0$

(d) $\iota \oplus_{G} O=1$

Generally, the conjunction $c$ obtained in this way is not symmetric, as suggested by the above example. In place of $i=\rightarrow_{*}$ we can use its contrapositive implication $i_{n}(x, y)=n(y) \rightarrow_{*} n(x)$. Its semi-dual conjunction is $c_{n}(x, y)=c(y, x)$ if $c$ is the semi-dual of $i[34,29]$. We can symmetrize these conjunctions considering $c^{\prime}(x, y)=\min (c(x, y), c(y, x))$ and its semi-dual implication. For instance

Example 3. Take the nilpotent minimum [34]:

$$
\overline{\min }(x, y)=\min \left(x \otimes_{G} y, y \otimes_{G} x\right)=\left\{\begin{array}{l}
\min (x, y) \text { if } x+y>1, \\
0 \text { otherwise }
\end{array}\right.
$$

for $c^{\prime}$ and the negation $n=1-$ on $[0,1]$. Let

$$
d(x, y)=\overline{\max }(x, y)=1-\overline{\min }(1-x, 1-y)=\left\{\begin{array}{l}
\max (x, y) \text { if } x+y<1 \\
1 \text { otherwise }
\end{array}\right.
$$

and the implication $i_{\min }(x, y)=\overline{\max }(1-x, y)=\max (1-x, y)$ if $x>y$, and 1 otherwise. Then one obtains the nilpotent min square of opposition choosing any pair of numbers $(\alpha, \epsilon)$ such that $\alpha+\epsilon \leq 1$ : 
(a) $\overline{\min }(\alpha, \epsilon)=0$

(b) $o=1-\alpha, \iota=1-\epsilon$

(c) $i_{\overline{\min }}(\alpha, 1-\epsilon)=1, i_{\overline{\min }}(\epsilon, 1-\alpha)=1$.

(d) $\overline{\max }(\iota, o)=1$

The same conditions for the square of opposition are obtained using Łukasiewicz connectives.

Example 4. [19] Take the Eukasiewicz conjunction, i.e., the conjunction $c_{L}$ defined by

$$
c_{L}(a, b)=\max (0, a+b-1),
$$

and the negation $1-(\cdot)$ on the scale $[0,1]$, one obtains the Eukasiewicz system leading to a square of opposition:

(a) $\alpha=1-o, \epsilon=1-\iota$

(b) $\min (1-\alpha+\iota, 1)=1, \min (1-\epsilon+o, 1)=1$ (using Eukasiewicz implication $\min (1,1-x+y))$.

(c) $\max (0, \alpha+\epsilon-1)=0$

(d) $\min (\iota+o, 1)=1$

This square is fully graded again for any pair of numbers $(\alpha, \epsilon)$ such that $\alpha+\epsilon \leq 1$ and leads to a graded square of opposition.

\subsection{The hexagon of opposition}

This section is a refresher on the work of Blanché $[16,15]$ that deals with the structural organization of concepts by means of the hexagon of opposition. He noticed that adding two vertices $\mathbf{U}$ and $\mathbf{Y}$, respectively defined as the disjunction of $\mathbf{A}$ and $\mathbf{E}$, and the conjunction of $\mathbf{I}$ and $\mathbf{O}$, to the square, a hexagon AUEOYI is obtained that contains 3 squares of opposition, AEOI, YAUO, and YEUI, each obeying the 4 properties enumerated above for the square.

Blanché [15] emphasized the point that the hexagonal picture can be found in many conceptual structures, such as for instance arithmetical comparators, or deontic modalities. He does not insist on the reason why this hexagonal structure is often encountered in very different topics. Such a hexagon is in fact obtained each time a tripartition of mutually exclusive situations such as $\mathbf{A}, \mathbf{E}$, and $\mathbf{Y}$ that play the same role is considered [25]. The tripartition underlying the hexagon was already mentioned by Sauriol [52].

Example 5. Let $U$ be the universe of discourse partitioned into three subsets $A, E$, and $Y$. We have $A \cap E=E \cap Y=A \cap Y=\emptyset, A \neq \emptyset, E \neq \emptyset, Y \neq \emptyset$ and $A \cup E \cup Y=U$. Hence we obtain the following hexagon of opposition:

Note that the requirement that the hexagon contains three squares of opposition implies that $A, E$, and $Y$ form a partition of $U$ (for instance already, $A$ and $E \cup Y$ are set-complements, expressing contradictories).

When, as in the above example, $A, E$, and $Y$ form a partition of $U$, Pellissier [46] speaks of a strong hexagon, while if $A \cup E \cup Y \subset U$, he speaks of a weak hexagon. 


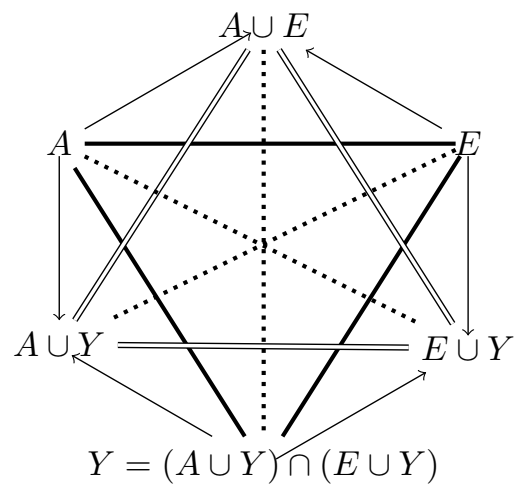

Figure 2. Hexagon induced by three disjoint sets $(A, E, Y)$

\subsection{The graded hexagon}

This subsection deals with a gradual extension of the strong hexagon of opposition [19, 32]. A graded hexagon of opposition is obtained by first assigning variables $\nu=d(\alpha, \epsilon)$ and $\gamma=c(\iota, o)$ to new vertices $\mathbf{U}$ and $\mathbf{Y}$. Then we must require additional conditions to ensure that YAUO and YEUI are proper squares of opposition playing the same role as AEOI Namely, we must have the following definition [19]:

Definition 3. A graded hexagon of opposition $\alpha \nu \epsilon o \gamma \iota$ is made of a graded square of opposition $\alpha \epsilon \mathrm{c}$ in the sense of Definition 2 plus the following conditions pertaining to the additional vertices

(e) $\mathbf{Y}$ and $\mathbf{U}$ are contradictories:

$$
\gamma=n(\nu)
$$

(f) Subaltern relations:

$$
i(\alpha, \nu)=i(\gamma, o)=i(\epsilon, \nu)=i(\gamma, \iota)=1 ;
$$

(g) Contrariety conditions:

$$
c(\alpha, \gamma)=c(\gamma, \alpha)=c(\gamma, \epsilon)=c(\epsilon, \gamma)=0
$$

(h) Subcontrariety conditions:

$$
d(\nu, o)=d(o, \nu)=d(\iota, \nu)=d(\nu, \iota)=1
$$

(i) Conditions linking vertices of the two squares YAUO and YEUI:

$$
\begin{aligned}
& \alpha=c(\iota, \nu)=c(\nu, \iota), \epsilon=c(\nu, o)=c(o, \nu) \\
& \iota=d(\alpha, \gamma)=d(\gamma, \alpha), o=d(\gamma, \epsilon)=d(\epsilon, \gamma) .
\end{aligned}
$$

The above equalities express additional relationships between $\alpha, \epsilon, o, \iota$. We obtain a graded hexagon if and only if these conditions are not conflicting. The conditions in the last item ensure that the three fuzzy sets in the tripartition induced by $(\alpha, \epsilon, \gamma)$ play the same role. As $\gamma$ and $\nu$ are contradictories, the above additional conditions for having a hexagon are not independent.

Proposition 3. Given an involutive negation $n: L \rightarrow L$, a triplet $(i, c, d)$ of implication, conjunction and disjunction defined as above, where the conjunction is commutative, and $\mathbf{Y}$ and $\mathbf{U}$ are contradictories, the conditions of 
Definition 3 reduce to

$$
c(\alpha, \gamma)=c(\gamma, \epsilon)=0 ; \quad \iota=d(\alpha, \gamma), o=d(\gamma, \epsilon)
$$

Proof Conditions $(h)$ are obtained from conditions $(g)$ by De Morgan duality. The first half of conditions $(i)$ is obtained from the second half by De Morgan duality; conditions $(f)$ are obtained from conditions $(g)$ by semi-duality.

If we drop conditions $(i)$ but preserve the contrariety ones, one may still consider that we have a hexagon of opposition, the gradual counterpart of what Pellissier calls weak hexagon. However, so-doing we implicitly admit that $(\alpha, \epsilon)$ are primitive while $\gamma$ is derivative, and they cannot be exchanged.

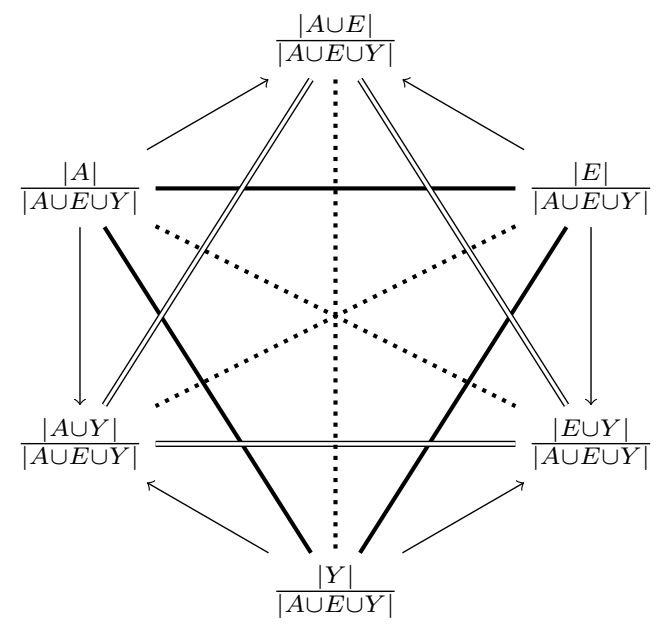

FIGURE 3. Graded hexagon with relative cardinalities

The following result is proved in [19] using the Łukasiewicz conjunction:

Proposition 4. If $n(x)=1-x$, and $c_{L}$ is the Eukasiewicz conjunction, and $i_{L}$ the Eukasiewicz implication, then the hexagon obtained from the triplet $\left(i_{L}, c_{L}, d_{L}\right)$ is a hexagon of opposition as soon as $\alpha+\epsilon \leq 1$.

In that case we have that $\gamma+\alpha+\epsilon=1$ which ensures a fuzzy tripartition (in the sense of Ruspini [51]) similar to the Boolean case. A general example of graded hexagon of opposition based on Łukasiewicz connectives is obtained by considering the relative cardinalities of the disjoint sets $A, E, Y$ in the hexagon of Figure 2. This is pictured on Figure 3

However the Kleene-Dienes setting does not allow for a graded hexagon.

Proposition 5. There is no weak graded hexagon of opposition using KleeneDienes triplet $(i(x, y), c(x, y), d(x, y))=(\max (1-x, y), \min (x, y), \max (x, y))$.

Proof Suppose $\alpha$ and $\epsilon$ are given. By definition of the hexagon, using KleeneDienes connectives, $u=\max (\alpha, \epsilon), \iota=1-\epsilon, o=1-\alpha$, and $\gamma=\min (\iota, o)=$ 
$\min (1-\alpha, 1-\epsilon)$. We must have that $\min (\alpha, \epsilon)=0$ as $\mathbf{A}$ and $\mathbf{E}$ are contradictories. Suppose $\alpha=0$, without loss of generality. Some of the previous conditions simplify into $u=\epsilon, o=1, \gamma=1-\epsilon$. By virtue of the weak hexagon, $\mathbf{Y}$ and $\mathbf{E}$ are contradictories, so $\min (\gamma, \epsilon)=0=\min (1-\epsilon, \epsilon)$. So $\epsilon \in\{0,1\}$, and other variables are thus Boolean as well. So there is no weak graded hexagon of opposition using Kleene-Dienes triplet.

Assume a commutative conjunction $c$ which yields a hexagon of opposition and a graded tripartition. Let us consider the additional conditions (GI). First if we assume that $c$ is such that $c(x, n(x))=0$ then the square condition on contraries $c(\alpha, \epsilon)=0$ implies $c(\alpha, \gamma)=c(\gamma, \epsilon)=0$ since $\gamma=c(\iota, o)=c(n(\epsilon), n(\alpha)) \leq \min (n(\epsilon), n(\alpha))$. So that it ensures $\alpha, \gamma$ and $\epsilon, \gamma$ to be contraries. Moreover if $c$ is such that $c(x, y)=0$ implies $c(n(x), n(c(n(x), n(y))))=y,{ }^{1}$ then it is clear that the other conditions in $(\mathrm{GI})$ are also satisfied. Indeed, for instance, $d(\alpha, \gamma)=n(c(n(\alpha), n(c(n(\epsilon), n(\alpha)))=$ $n(\epsilon)$. In other words,

Proposition 6. If the commutative conjunction $c$ and negation $n$ are such that $c(x, n(x))=0$, and moreover $c(x, y)=0$ implies $c(n(x), n(c(n(x), n(y))))=$ $y$, then $\alpha \nu \epsilon O \gamma \iota$ forms a hexagon of opposition.

We can choose a continuous triangular norm [40] for $c$. Suppose its De Morgan dual $d$ is of the form $d(x, y)=s^{-1}(\min (1, s(x)+s(y)))$, where $s$ is any continuous strictly increasing function from $[0,1]$ to $[0,1]$ with $s(0)=$ $0, s(1)=1$. Suppose $n$ is such that $s(n(x))=1-s(x)$. Then $c(x, y)=$ $s^{-1}(\max (0, s(x)+s(y)-1))$. We have a hexagon of opposition that extends the Łukasiewicz case. Namely $c(x, n(x))=0$ since $s(c(x, n(x)))=\max (0, s(x)+$ $1-s(x)-1)=0$ and $c(n(x), d(x, y))=s^{-1}(\max (0,1-s(x)+\min (1, s(x)+$ $\left.s(y))-1))=s^{-1}(\min (1-s(x), s(y)))\right)=y$ since $c(x, y)=0$ comes down to $s(x)+s(y) \leq 1$. The hexagon uses $\alpha, \epsilon, \gamma$ with $s(\alpha)+s(\epsilon)+s(\gamma)=1$.

It seems difficult to extend the square of opposition of Example 2 to a graded hexagon of opposition. The reason is that enforcing the conditions in Proposition 6 leads to an impossibility. Indeed the condition $c(x, 1-x)=0$ is satisfied by $c=\otimes_{G}$, but the condition $c(n(x), n(c(n(x), n(y))))=y$ if $c(x, y)=0$ is more problematic. It reads $(1-x) \otimes_{G}\left(x \oplus_{G} y\right)=y$ if $x+y \leq 1$. In that case $(1-x) \otimes_{G}\left(x \oplus_{G} y\right)=(1-x) \otimes_{G} y$, which is equal to $y$ only if $x<y$. So, it means that in conditions (GI), the condition $\iota=d(\alpha, \gamma)$ requires $\alpha<\epsilon$ and the condition $o=d(\gamma, \epsilon)$ requires $\alpha>\epsilon$, which is a contradiction.

A similar problem occurs with Example 3. Assume $\alpha+\epsilon \leq 1$ again. If $\alpha+\epsilon<1$, the $\mathbf{U}$ corner is labeled by $\overline{\max }(\alpha, \epsilon)=\max (\alpha, \epsilon)$ and the $\mathbf{Y}$ corner is labeled by $\overline{\min }(1-\alpha, 1-\epsilon)=\min (1-\alpha, 1-\epsilon)$. To get a hexagon, we need the following conditions:

- on $\mathbf{A}$ and $\mathbf{Y}: \overline{\min }(\alpha, \min (1-\alpha, 1-\epsilon))=0 ; \overline{\max }(\alpha, \min (1-\alpha, 1-\epsilon)=1-\epsilon$, which requires $\epsilon \geq \alpha$.

\footnotetext{
${ }^{1}$ In the crisp case it means $\bar{A} \cap(A \cup B)=B$ if $A$ and $B$ are disjoint.
} 
- on $\mathbf{E}$ and $\mathbf{Y}: \overline{\min }(\epsilon, \min (1-\alpha, 1-\epsilon))=0 ; \overline{\max }(\epsilon, \min (1-\alpha, 1-\epsilon)=1-\alpha$, which requires $\epsilon \leq \alpha$.

So we need that $\alpha=\epsilon<0.5$ to get a graded hexagon. But, we also need $\alpha=c(\iota, \nu)$, i.e., $\alpha=c(\alpha, 1-\alpha)=0$. So, we only recover the Boolean hexagon.

If $\alpha+\epsilon=1$, then $\overline{\max }(\alpha, \epsilon)=1$ for vertex $\mathbf{U}$, and $\overline{\min }(1-\alpha, 1-\epsilon)=0$ for vertex $\mathbf{Y}$. In this case, $\overline{\max }(\alpha, \overline{\min }(1-\alpha, 1-\epsilon))=0 \neq 1-\epsilon$, except if $\epsilon=1$ and $\alpha=1$. So, we only recover the Boolean hexagon in this case too.

\subsection{Cubes of opposition}

As pointed out by Béziau [8] there is no cube, each facet of which is a square of opposition. Nevertheless, we encounter in the literature various cubic structures of oppositions that generalize the square of opposition. Two cubes are particularly worth of interest, Moretti's cube [42, 43] and Dubois-Prade's cube [25]. They are presented in this subsection, leaving apart the analogical cube of opposition $[4,5]$ since it contains no traditional square of opposition. We begin with the cube proposed by Moretti [43].

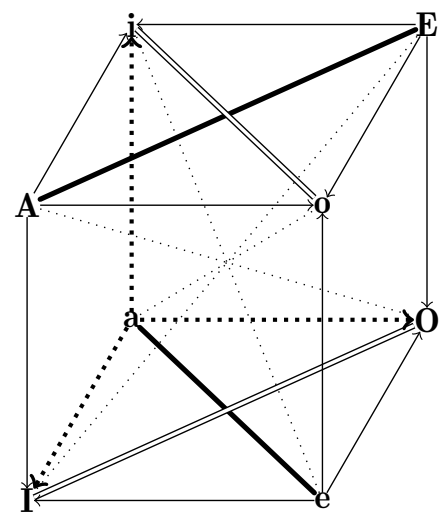

FiguRE 4. Moretti's cube of opposition

2.5.1. The cube of opposition proposed by A. Moretti. Moretti [42, 43] has introduced a cube of opposition where all edges are entailments, and which relies on four mutually exclusive situations whose elements are the four vertices from which the entailments start. The structure is pictured in Figure 4, where the eight vertices $\mathbf{A}, \mathbf{E}, \mathbf{O}, \mathbf{I}, \mathbf{a}, \mathbf{e}, \mathbf{o}, \mathbf{i}$ are named in a square of opposition manner. Indeed, this cube can be obtained from two squares of opposition AEOI and aeoi (the two squares can be seen in Figure 4, where the different edges and diagonals follow the conventions used in this paper (thick line for mutual exclusiveness, dotted lines for contradictories, etc.). It ensures that the vertical edges are entailments. For making sure that the other entailments hold, we have to impose the following constraint

$$
(\mathbf{A} \vee \mathbf{E}) \rightarrow(\mathbf{i} \wedge \mathbf{o}),
$$


which summarizes the four entailments of the top facet; since $\mathbf{A} \equiv \neg \mathbf{O}$, $\mathbf{E} \equiv \neg \mathbf{I}, \mathbf{a} \equiv \neg \mathbf{O}, \mathbf{e} \equiv \neg \mathbf{i}$, this entails that $(\mathbf{a} \vee \mathbf{e}) \rightarrow(\mathbf{I} \wedge \mathbf{O})$ holds as well, which guarantees the four entailments of the bottom facet. As a consequence, $(\mathbf{A} \vee \mathbf{E}) \rightarrow(\neg \mathbf{a} \wedge \neg \mathbf{e})$ also holds, since $\neg(\mathbf{a} \vee \mathbf{e}) \equiv \mathbf{i} \wedge \mathbf{o}$; this expresses that $\mathbf{A}$, $\mathbf{E}, \mathbf{a}$, and $\mathbf{e}$ are mutually exclusive (since we also have the square conditions $\neg \mathbf{A} \vee \neg \mathbf{E}$ and $\neg \mathbf{a} \vee \neg \mathbf{e}$ by construction).

Thus this cube of opposition is based on four mutually exclusive vertices, corresponding to the four vertices $\mathbf{A}, \mathbf{E}, \mathbf{a}, \mathbf{e}$ from which arrows leave, while the four vertices where the arrows arrive are $\mathbf{I}, \mathbf{O}, \mathbf{i}, \mathbf{o}$. This cube of opposition contains 6 squares of opposition in the diagonal plans of the cube: AEOI, AaOo, AeOi, aEoI, eEiI and aeoi, as can be easily checked. Each square is obtained by taking two vertices among $\mathbf{A}, \mathbf{E}, \mathbf{a}, \mathbf{e}$, together with their two contradictories, which indeed leaves $\left(\begin{array}{l}4 \\ 2\end{array}\right)=6$ possibilities. Obviously each of these squares of opposition can be completed by a hexagon of opposition.

Not all squares of opposition appear on Figure 4 in order not to overload it. However we can complete it by expressing contraries (boldface line) on diagonals Ee, Aa, Ae, aE of facets of the cube, and subcontraries (double lines) on diagonals $\mathbf{I i}, \mathbf{o O}, \mathbf{O i}, \mathbf{o I}$.

Conversely, starting with four pairwise disjoint subsets $A, E, A^{\prime}, E^{\prime}$ that make a tetrahedron, one can easily obtain Moretti's cube, by associating the union of the three other subsets to the vertex diagonally opposed to each of the vertices $A, E, A^{\prime}, E^{\prime}$, as can be seen on Figure 5. Thus the twelve edges correspond to entailments that hold. It is worth noticing that is not required that $A, E, A^{\prime}, E^{\prime}$ make a partition, indeed there is no requirement on the set $A \cup E \cup A^{\prime} \cup E^{\prime}$, i.e., $T=\bar{A} \cap \bar{E} \cap \overline{A^{\prime}} \cap \overline{E^{\prime}}$ is not necessarily empty. In other words, there may exist elements such that $\mathbf{I} \wedge \mathbf{O} \wedge \mathbf{i} \wedge \mathbf{o}$, i.e., $\mathbf{Y} \wedge \mathbf{y}$ is true (where $\mathbf{A}, \mathbf{E}, \mathbf{Y}$ and $\mathbf{a}, \mathbf{e}, \mathbf{y}$ are the tri-partitions we start with on hexagons). In this sense, Moretti's cube is closer in spirit to the square of opposition (two disjoint sets not forming a partition) or the weak hexagon (three disjoint sets not forming a partition) than to the strong hexagon.

It seems there are fewer natural examples of four disjoint sets not forming a partition than of two disjoint sets as involved in the square. But there are examples of Moretti's cube where the four sets form a partition (they may be considered as degenerated in the sense that they are not based on a 5 -set partition). One is obtained when we compare two objects $x$ and $y$ by means of a reflexive outranking relation for which " $x$ is not comparable to $y$ " is an option together with " $x$ is preferred to $y$ ", " $y$ is preferred to $x$ ", and "one is indifferent between $x$ and $y$ " [50], as already pointed out in [25]. We shall use this remark in the last section of this paper. Another example is obtained from the partition induced by two propositional variables.

Example 6. A basic example of Moretti's cube is obtained by starting from the partition induced by conjunctions of literals formed with two propositional variables: $p \wedge q, p \wedge \neg q, \neg p \wedge q$ and $\neg p \wedge \neg q$. In this case, $\mathbf{I} \wedge \mathbf{O} \wedge \mathbf{i} \wedge \mathbf{o}$, i.e., $\mathbf{Y} \wedge \mathbf{y}$ is a contradiction: Indeed $\mathbf{Y}$ corresponds to $\neg p$ and $\mathbf{y}$ to $p$, as can be 


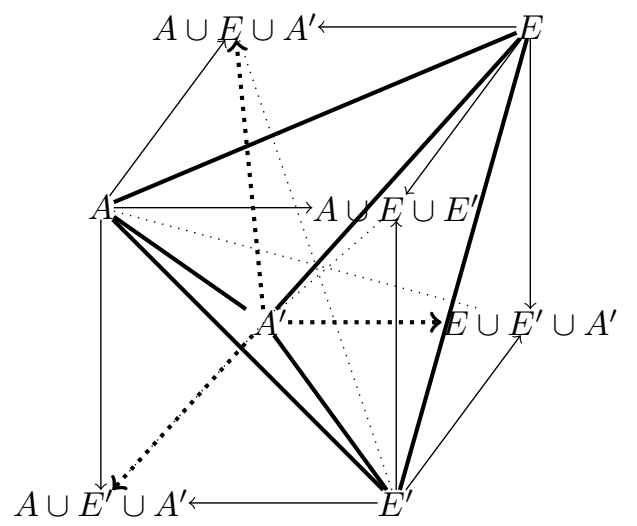

Figure 5. Moretti's cube of opposition induced by four disjoint sets $\left(A, E, A^{\prime}, E^{\prime}\right)$

checked on Figure 6. This logical cube is a part of a more general structure exhibiting the 16 binary Boolean connectives [41, 44, 47, 25].

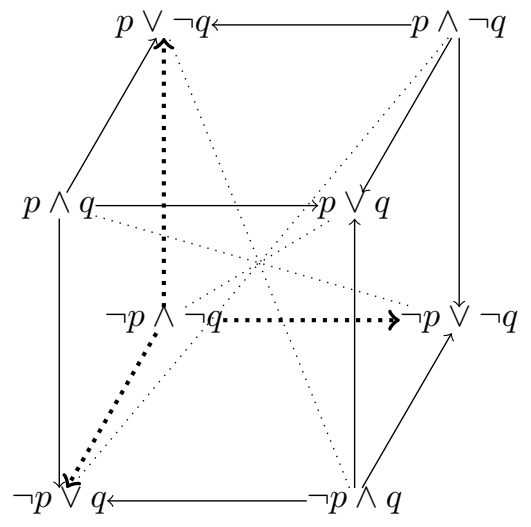

FiguRE 6. Moretti's cube of opposition with logical connectives

2.5.2. Gradual extension of Moretti's cube. Let us assume that $\alpha, \epsilon, o, \iota$, and $\alpha^{\prime}, \epsilon^{\prime}, o^{\prime} \iota^{\prime}$ denote the grades associated to vertices A, E, O I, and a, e, $\mathbf{o}$ i. Considering an involutive negation $n$, a conjunction $c$, an implication $i$ linked by semi-duality and $d$ a disjunction associated by De Morgan duality we can define a graded Moretti's cube as follows:

Definition 4. A graded cube of opposition in the sense of Moretti is defined by attaching $L$-valued variables $\alpha, \epsilon, o, \iota \alpha^{\prime}, \epsilon^{\prime}, o^{\prime}$ and $\iota^{\prime}$ to vertices $\mathbf{A}, \mathbf{E}, \mathbf{O}$, I, a, e o, i respectively, in such a way that: 
- AEOI and aeoi are two graded squares of opposition in the sense of Definition 2.

- The following entailments on the top and bottom facets AEoi and aeOI hold:

(j) $i\left(\alpha, \iota^{\prime}\right)=1, i\left(\alpha, o^{\prime}\right)=1, i\left(\epsilon, \iota^{\prime}\right)=1, i\left(\epsilon, o^{\prime}\right)=1$,

(k) $i\left(\alpha^{\prime}, \iota\right)=1, i\left(\alpha^{\prime}, o\right)=1, i\left(\epsilon^{\prime}, \iota\right)=1, i\left(\epsilon^{\prime}, o\right)=1$.

Property $(j)$ is the counterpart of the condition $(\mathbf{A} \vee \mathbf{E}) \rightarrow(\mathbf{i} \wedge \mathbf{o})$ in the Boolean case since it can be written as $i\left(\max (\alpha, \epsilon), \min \left(\iota^{\prime}, o^{\prime}\right)\right)=1$.

Proposition 7. The graded Moretti's cube of opposition contains 6 squares of opposition: AEOI, aeoi, AaOo, AeOi, aEoI and eEiI.

Proof: AaOo is a square of opposition: $\alpha$ and $o$ (resp. $\alpha^{\prime}$ and $o^{\prime}$ ) are each other's negation because AEOI (resp. aeoi) are two graded squares of opposition.

Subaltern relations between $\alpha$ and $o^{\prime}$ (resp. $\alpha^{\prime}$ and $o$ ) hold: $i\left(\alpha, o^{\prime}\right)=1$ and $i\left(\alpha^{\prime}, o\right)=1$ because of the entailments condition on the top and bottom facets. Mutual exclusion conditions between $\alpha$ and $\alpha^{\prime}, c\left(\alpha, \alpha^{\prime}\right)=n\left(i\left(\alpha, o^{\prime}\right)\right)=$ 0 , hold because of the entailments condition on the top and bottom facets. Finally $d\left(o, o^{\prime}\right)=n\left(c\left(\alpha, \alpha^{\prime}\right)\right)=1$. For AeOi, aEoI and eEiI, the proof is similar to the previous one.

An additional property of the implication defining the subaltern conditions enables the number of conditions to make a Moretti cube to be reduced.

Proposition 8. If the implication is such that $i(x, y)=1$ if and only if $i(n(y), n(x))=1$ then a graded Moretti's cube of opposition is defined by two graded squares of opposition AEOI and aeoi associated with the entailments on the top facet or the bottom facet.

Proof: The additional property entails that the subaltern relations on the top facet imply the ones on the bottom facet and conversely: If this additional property of implication $i$ holds, the condition $(k)$ in Definition 4 follows from $(j)$.

Example 7. Consider four values $\alpha+\epsilon+\epsilon^{\prime}+\alpha^{\prime} \leq 1$ and the Eukasiewicz conjunction $c_{L}$ (eq. 1), the implication associated by semi-duality $i_{L}(x, y)=$ $\min (1,1-x+y)$, the duality associated by De-Morgan duality $d_{L}(x, y)=$ $\min (1, x+y)$ where $1-(\cdot)$ denotes an involutive negation. The graded cube of opposition defined by the following two graded squares of opposition:

- AEOI with $\mathbf{A}: \alpha, \mathbf{E}: \epsilon, \mathbf{O}: 1-\alpha$ and $\mathbf{I}: 1-\epsilon$,

- aeoi with $\mathbf{a}: \alpha^{\prime}$, e : $\epsilon^{\prime}, \mathbf{o}=1-\alpha^{\prime}$ and $\mathbf{i}=1-\epsilon^{\prime}$

is a graded Moretti's cube of opposition.

It is easy to check that AEOI and aeoi are squares of opposition. We have $i_{L}(x, y)=i_{L}(n(y), n(x))$ hence we just need to check the entailment conditions on the top facet. We have 


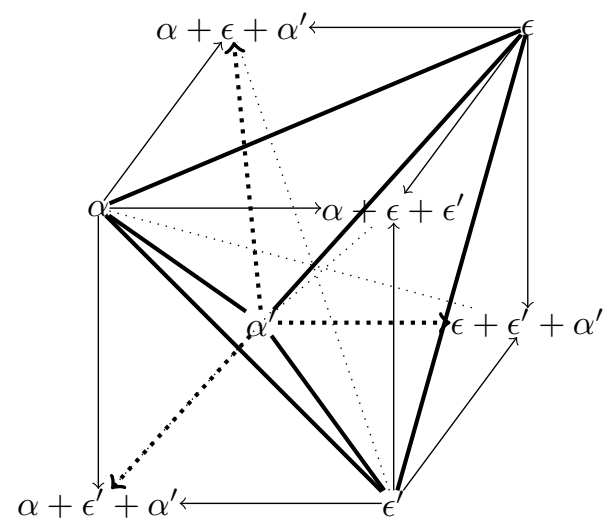

Figure 7. Graded version of Moretti's cube of opposition induced by four numbers $\left(\alpha+\epsilon+\epsilon^{\prime}+\alpha^{\prime} \leq 1\right)$

- $\mathbf{A} \rightarrow \mathbf{i}: i_{L}\left(\alpha, 1-\epsilon^{\prime}\right)=\min \left(1,1-\alpha+1-\epsilon^{\prime}\right)=1$

- $\mathbf{A} \rightarrow \mathbf{o}: i_{L}\left(\alpha, 1-\alpha^{\prime}\right)=\min \left(1,1-\alpha+1-\alpha^{\prime}\right)=1$

- $\mathbf{E} \rightarrow \mathbf{i}: i_{L}\left(\epsilon, 1-\epsilon^{\prime}\right)=\min \left(1,1-\epsilon+1-\epsilon^{\prime}\right)=1$

- $\mathbf{E} \rightarrow \mathbf{o}: i_{L}\left(\epsilon, 1-\alpha^{\prime}\right)=\min \left(1,1-\epsilon+1-\alpha^{\prime}\right)=1$

This is a graded version of the set-based cube of Figure 5.

In the above example, the condition $\alpha+\epsilon+\epsilon^{\prime}+\alpha^{\prime} \leq 1$ is too strong. It is enough to have 6 conditions of the form $x+y \leq 1$ for $x \neq y \in\left\{\alpha, \alpha^{\prime}, \epsilon, \epsilon^{\prime}\right\}$, (which means $\alpha+\epsilon \leq 1, \alpha^{\prime}+\epsilon^{\prime} \leq 1$ and $\max \left(\epsilon^{\prime}, \alpha^{\prime}\right) \leq \min (1-\alpha, 1-\epsilon)$ ) to get the 6 graded squares of opposition in Moretti's cube. This result remains true, if instead of Lukasiewicz system, we use a more general triplet $(c, i, d)$ with conjunction such that $c(x, y)=0$ if and only if $x \leq n(y)$ (for instance, the nilpotent minimum), or with implication $i$ such that $i(x, y)=1$ if and only if $x \leq y$ (as per Proposition 2).

The above graded Moretti cube with Łukasiewicz connectives can be examplified using cardinalities of sets of objects $X$ and $Y$ satisfying two Boolean properties $p$ and $q$ respectively, making the cube of Fig. 6 gradual. We assume $X \neq \emptyset, \bar{X} \neq \emptyset, Y \neq \emptyset, \bar{Y} \neq \emptyset$. Thus $X \cap Y, \bar{X} \cap Y, X \cap \bar{Y}, \bar{X} \cap \bar{Y}$ form a quadri-partition of the set $U$ of objects (as none of them are empty), and we obtain the cube of Figure 8, which is a graded counterpart of the one of Figure 6.

In contrast, if we use a conjunction $c$ without zero divisors, such as min and product, the graded Moretti cube almost degenerates into a Boolean one. Indeed, the implication $i$ is then such that $i(x, y)=1$ if and only if $x=0$ or $y=1$. Then the 6 above conditions read $\alpha=0$ or $\epsilon=0, \alpha^{\prime}=0$ or $\epsilon^{\prime}=0$ and $\max \left(\epsilon^{\prime}, \alpha^{\prime}\right)=0$ or $\min (n(\alpha), n(\epsilon))=1$. Suppose $1>\alpha>0$. It implies $\epsilon=0$ and $0<\min (n(\alpha), n(\epsilon))<1$ so $\epsilon^{\prime}=\alpha^{\prime}=0$. By symmetry, the case where $0<\epsilon<1$ entails $\epsilon^{\prime}=\alpha^{\prime}=\alpha=0$. In other words, graded Moretti's cubes with such conjunctions are degenerated ones with only two 


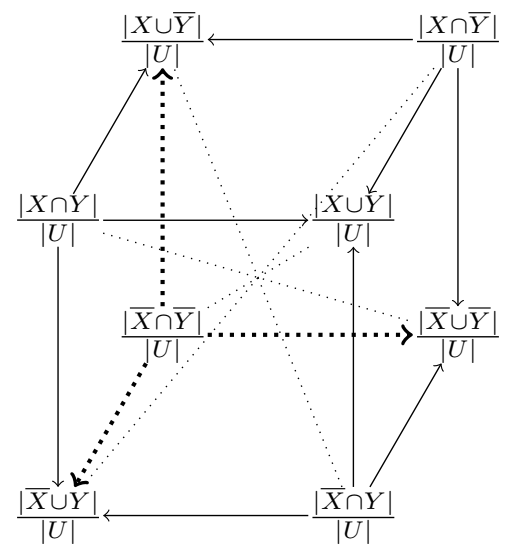

FIGURE 8. Moretti's cube of opposition with similarity indices

opposite non-Boolean vertices (e.g., $\mathbf{A}$ valued with $\alpha$ and $\mathbf{O}$ valued with $n(\alpha)$ on Figure 4). For instance this is what happens with Kleene-Dienes Moretti's cube, using $c=\min$ (extending Example 1).

2.5.3. Graded Moretti logical cubes. Finally we can also generalize both cubes of Figures 6 and 7 using two many-valued propositional variables say $x$ and $y$ on $[0,1]$, replacing the Boolean $p$ and $q$. Define conjunction of $x$ and $y$ by product $x y$, and negation by $1-\cdot$. Note that the product is the only t-norm $\odot$ such that $x \odot y+x \odot(1-y)=x[3]$. Then we can define the parameters on the cube of Figure 7 as follows:

$$
\alpha=x y ; \epsilon=x(1-y) ; \alpha^{\prime}=(1-x)(1-y) ; \epsilon^{\prime}=(1-x) y
$$

And we can check that all vertices of the Lukasiewicz cube of Figure 7 can be expressed as a gradual version of the logical cube of Figure 6 :

- $\alpha+\epsilon+\epsilon^{\prime}=1-(1-x)(1-y)=x+y-x y$ (corresponding to the conorm $x \oplus y$ dual to the product, expressing gradual disjunction)

- $\alpha+\epsilon+\alpha^{\prime}=x \oplus(1-y)$

- $\alpha^{\prime}+\epsilon+\epsilon^{\prime}=(1-x) \oplus(1-y)$

- $\alpha+\epsilon^{\prime}+\alpha^{\prime}=(1-x) \oplus y$

- Moreover $\alpha+\alpha^{\prime}+\epsilon+\epsilon^{\prime}=1$

This case can even be generalized if we assume that $x \odot y+x \odot{ }^{\prime}(1-y)=x$ for distinct t-norms $\odot$ and $\odot$, and we still assume that the De Morgan dual $\oplus$ of $\odot$ satisfies $x \oplus y=1-(1-x) \odot(1-y)=x+y-x \odot y$ (a gradual counterpart of the cardinality property $|A \cup B|+|A \cap B|=|A|+|B|)$. This implies that $x+y-x \odot y$ should be associative, which imposes the existence of a real-valued parameter $s>0$ characterizing operation $\odot=\odot_{s}$ as follows:

$$
x \odot_{s} y=\log _{s}\left(1+\frac{\left(s^{x}-1\right)\left(s^{y}-1\right)}{s-1}\right) .
$$


This is a result due to Frank [36], and $\odot_{s}$ is called Frank t-norm. We have that $\lim _{s \rightarrow 0} x \odot_{s} y=\max (0, x+y-1), \lim _{s \rightarrow+\infty} x \odot_{s} y=\min (x, y)$ and $\odot_{1}$ is the product. In this case, we can check [21] that $\odot^{\prime}=\odot_{1 / s}$, i.e., $x \odot_{s} y+$ $x \odot_{1 / s}(1-y)=x$. The De Morgan dual of $\odot_{s}$ is the co-norm [40]:

$$
x \oplus_{s} y=1-\log _{s}\left(1+\frac{\left(s^{1-x}-1\right)\left(s^{1-y}-1\right)}{s-1}\right) .
$$

Proposition 9. Choosing parameters on the Eukasiewicz cube of Figure 7 as follows:

$$
\alpha=x \odot_{s} y ; \epsilon=x \odot_{1 / s}(1-y) ; \alpha^{\prime}=(1-x) \odot_{s}(1-y) ; \epsilon^{\prime}=(1-x) \odot_{1 / s} y
$$

we can check that all vertices of the Eukasiewicz cube of Figure 7 can be expressed as:

- $\alpha+\epsilon+\epsilon^{\prime}=1-(1-x) \odot_{s}(1-y)=x \oplus_{s} y$

- $\alpha+\epsilon+\alpha^{\prime}=1-(1-x) \odot_{1 / s} y=x \oplus_{1 / s}(1-y)$

- $\alpha^{\prime}+\epsilon+\epsilon^{\prime}=1-x \odot_{s} y=(1-x) \oplus_{s}(1-y)$

- $\alpha+\epsilon^{\prime}+\alpha^{\prime}=1-x \odot_{1 / s}(1-y)=(1-x) \oplus_{1 / s} y$

- Moreover $\alpha+\alpha^{\prime}+\epsilon+\epsilon^{\prime}=1$

Proof: Consider the first identity:

$\alpha+\epsilon+\epsilon^{\prime}=x \odot_{s} y+x \odot_{1 / s}(1-y)+(1-x) \odot_{1 / s} y=x+(1-x) \odot_{1 / s} y=$ $x+y-x \odot_{s} y=x \oplus_{s} y$. The other identities are proved in the same way.

Another interpretation of $x$ and $y$ on vertices could be probabilities $x=P(p)$ and $y=P(q)$ of logically independent Boolean propositions $p$ and $q$. Then the property $P(p \wedge q)=P(p) \odot_{s} P(q)$ expresses a parameterized form of probabilistic dependence, the case $s=1$ corresponding to the stochastic independence, and the additivity property of probability being ensured by the fact that $P(p) \odot_{s} P(q)+P(p) \odot_{1 / s} P(\neg q)=P(p)[21]$.

2.5.4. The cube of opposition proposed by Dubois and Prade. The cube of opposition rediscovered in [25,31] apparently appears for the first time in books by two XIXth century logicians, i.e. W. E Johnson [38] and J. N. Keynes [39], discussing syllogisms involving sentences of the form "all $P$ 's are $Q$ 's" and their related forms using negation. In such a cube (actually represented by these authors in the shape of an octagon), the front facet and the back facet are squares of opposition, and the style of edges follows the conventions of Figure 1 for contraries, subcontraries, subalterns and contradictories. As a summary, such a cube of opposition, we call $J K$-cube in the following, obeys the following requirements. ${ }^{2}$

Definition 5. Let A, I, E, O, a, i, e, o be propositional variables. In a JK-cube of opposition AEOIaieo, the following relations are supposed to hold:

\footnotetext{
${ }^{2}$ In previous papers $[18,19,31]$, we wrongly credited Reichenbach [49] for inventing this cube in the setting of syllogisms, because we omitted to consider additional constraints of non-equality of involved predicates introduced by him. This point is discussed later on in the next subsection.
} 
- Front and back facets: AEOI and aeoi are two squares of opposition in the sense of Definition 1.

- Side facets: subaltern relations (entailments):

(l) $\neg \mathbf{A} \vee \mathbf{i} ; \neg \mathbf{a} \vee \mathbf{I} ; \neg \mathbf{e} \vee \mathbf{O} ; \neg \mathbf{E} \vee \mathbf{o}$

- Top and bottom facets:

(m) $\mathbf{a}$ and $\mathbf{E}$ are contraries, i.e., $\neg \mathbf{a} \vee \neg \mathbf{E}$; the same for $\mathbf{A}$ and $\mathbf{e}$, i.e., $\neg \mathbf{A} \vee \neg \mathbf{e} ;$

(n) $\mathbf{i}$ and $\mathbf{O}$ are sub-contraries, i.e., $\mathbf{i} \vee \mathbf{O}$; the same for $\mathbf{I}$ and $\mathbf{o}$, i.e., $\mathbf{I} \vee \mathbf{o}$.

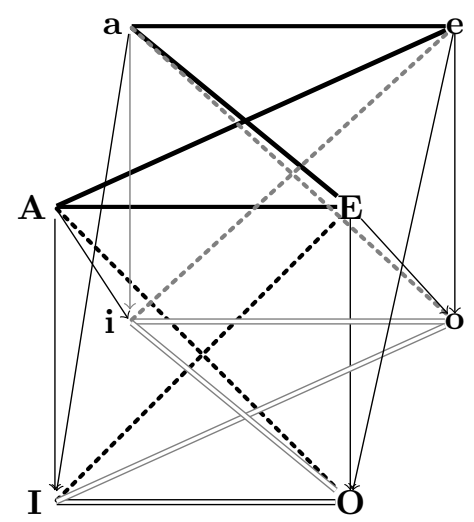

FiguRE 9. JK cube of opposition

Due to the conditions in Definition 5, it is clear that there are two more squares of opposition in the JK cube: AeOi, aEoI. Note they are twisted, non-planar squares.

What we call here "JK cube" was in fact presented as an octagon by Johnson [38] and Keynes [39]. The octagon counterpart of the cube of Figure 9 is shown in Figure 10, using visual conventions for lines as in the former figure. The same relations as in the cube can be found between the 8 vertices. ${ }^{3}$ In particular, the 4 squares of opposition are easy to identify.

The conditions that define the cube are redundant $[19,31]$. For a JK cube of opposition, the following properties hold:

- the properties on the front and back facets associated with the properties on the side facets entail the properties on the top and bottom facets.

\footnotetext{
${ }^{3}$ The actual octagon of Johnson and Keynes does not materialize the bold lines expressing mutual exclusiveness, while other lines relating $\mathbf{A}$ to $\mathbf{a}$ and $\mathbf{E}$ to $\mathbf{e}$ appear and are labeled "complementaries"; likewise, lines relating $\mathbf{I}$ to $\mathbf{i}$ and $\mathbf{O}$ to $\mathbf{o}$ appear and are labeled "subcomplementaries". For instance, $\mathbf{A}=$ all $P$ 's are $Q$ and $\mathbf{a}=$ all $Q$ 's are $P$ complement each other in the sense that if they both hold, $P$ and $Q$ are identical. Interestingly, in the Reichenbach cube, both $\mathbf{A}$ and $\mathbf{a}$, and $\mathbf{E}$ and $\mathbf{e}$, are mutually exclusive (and called "opposite"), since $P$ is supposed not to be equal to $Q$. Moreover yet other lines relating $\mathbf{A}$ to $\mathbf{o}, \mathbf{E}$ to $\mathbf{i}, \mathbf{O}$ to $\mathbf{a}$ and $\mathbf{I}$ to $\mathbf{e}$ are labeled "contra-complementaries". Observe that these different forms of complementarity do not appear in the traditional square.
} 


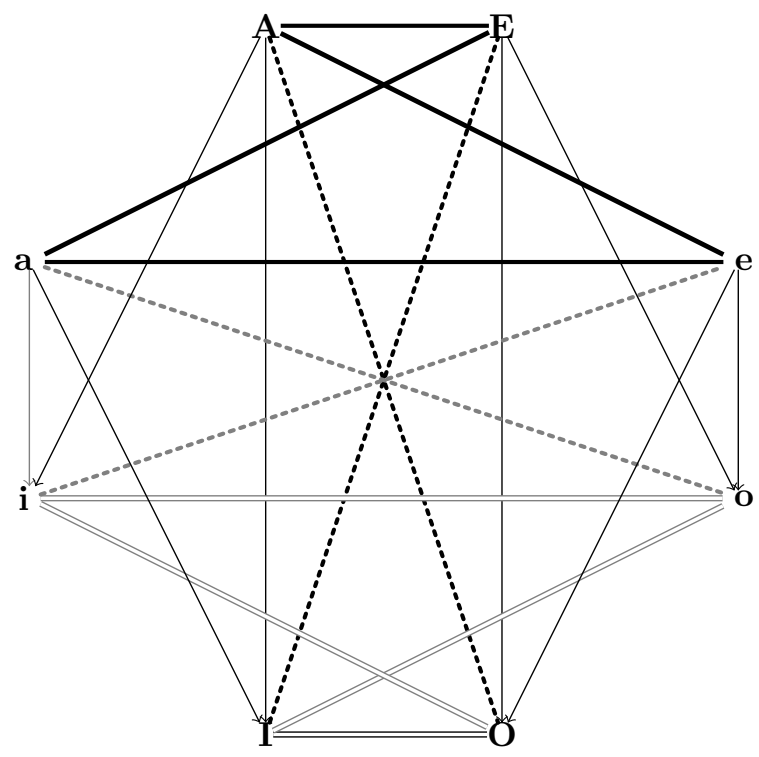

Figure 10. Johnson-Keynes octagon

- the properties on the front and back facets associated with the properties on the top (resp. bottom) facet entail the properties on the side facets and of the bottom (resp. top) facet.

A stronger result has been shown in [31]:

Proposition 10. The JK cube $\mathbf{A}, \mathbf{E}, \mathbf{I}, \mathbf{O}, \mathbf{a}, \mathbf{e}, \mathbf{i}, \mathbf{o}$ is characterized by the condition

$$
(\mathbf{A} \vee \mathbf{a}) \rightarrow(\neg \mathbf{E} \wedge \neg \mathbf{e}) .
$$

where vertices $\mathbf{I}, \mathbf{O}, \mathbf{i}, \mathbf{O}$ are then defined as contradictories: $\mathbf{I} \equiv \neg \mathbf{E}, \mathbf{O} \equiv \neg \mathbf{A}, \mathbf{i} \equiv \neg \mathbf{e}$, $\mathbf{o} \equiv \neg \mathbf{a}$.

The above condition indeed expresses that the pairs $(\mathbf{A}, \mathbf{E}),(\mathbf{A}, \mathbf{e}),(\mathbf{a}, \mathbf{E}),(\mathbf{a}, \mathbf{e})$ are mutually exclusive. Interestingly, the condition in Proposition 10 that characterizes the JK cube has a structure that is similar to the one that defines Moretti's cube, but this condition does not involve the same vertices. However we can show the following result.

Proposition 11. The JK cube is more general, i.e. less constrained, than Moretti's cube.

Proof: In Moretti's cube the condition $(\mathbf{A} \vee \mathbf{E}) \rightarrow(\mathbf{i} \wedge \mathbf{o})$ holds. Since $\mathbf{i} \equiv \neg \mathbf{e}, \mathbf{o} \equiv \neg \mathbf{a}$, it also reads $(\mathbf{A} \vee \mathbf{E}) \rightarrow(\neg \mathbf{e} \wedge \neg \mathbf{a})$. Thus $\mathbf{A} \rightarrow \neg \mathbf{e}$ and $\mathbf{E} \rightarrow \neg \mathbf{a}$. But we also have $\mathbf{A} \rightarrow \neg \mathbf{E}$ and $\mathbf{a} \rightarrow \neg \mathbf{e}$ in Moretti's cube (mutual exclusiveness conditions). Thus, we get $(\mathbf{A} \vee \mathbf{a}) \rightarrow(\neg \mathbf{E} \wedge \neg \mathbf{e})$, which is the characteristic condition of the JK cube. In Moretti's cube, two additional constraints 
hold: $\mathbf{A} \rightarrow \neg \mathbf{a}$ and $\mathbf{E} \rightarrow \neg \mathbf{e}$.

To get Moretti's cube from the JK-cube, it is enough to add bold-faced lines Aa and Ee (expressing contraries) to the JK cube, and double lines Oo and Ii (expressing subcontraries), and reposition the vertices of the latter accordingly. It corresponds to add the squares of opposition AaOo and eEiI.

The JK cubes of opposition may be found in many settings including 1st order classical logic [39], modal logics under relational semantics [19], formal concept analysis [25], rough set theory [18] and abstract argumentation [1].

2.5.5. Reichenbach's cube. In a paper from the mid-XXth century rediscussing syllogisms, Reichenbach [49] proposes yet another cube which turns out to be isomorphic to Moretti's. Namely, starting from the JK-cube, we first exchange the vertices a and $\mathbf{e}$, and $\mathbf{i}$ and $\mathbf{o}$, respectively, as in Figure 11.

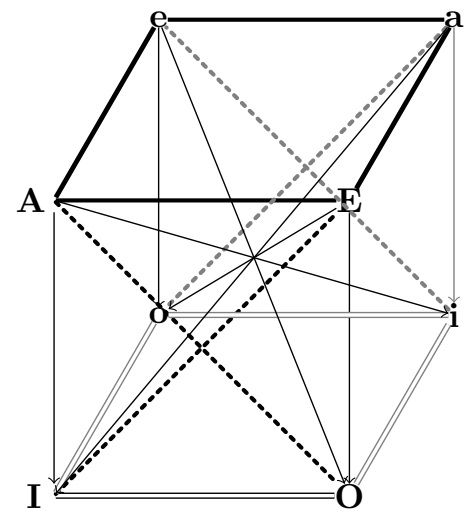

Figure 11. Reichenbach cube of opposition

Reichenbach puts additional constraints on this cube, namely that a and A, as well as E and e should be mutually exclusive (he calls them "opposite"), adding two diagonals in bold lines on the top square of the cube in Fig. 11, and the consequent double, dotted and directed lines, we omit to visualize for preserving legibility. These additional requirements come down to adding the constraint $(\mathbf{A} \vee \mathbf{E}) \rightarrow(\neg \mathbf{a} \wedge \neg \mathbf{e})$, which makes it clear that Reichenbach's cube is isomorphic to Moretti's cube up to a repositoning of the vertices. Note that the induced additional squares of opposition AaOo, and eEiI are not situated on a plane in Reichenbach's cube.

In terms of syllogisms, node A expresses that "all $P$ 's are $Q$ ", and the node a expresses that "all $Q$ 's are $P$ ". The additional condition demands that these conditions are not true together, which enforces that $P$ is not equal to $Q$ (indeed, a and $\mathbf{A}$ express the same statement only if $P=Q$ ). Likewise, node e expresses that " all not- $P$ 's are $Q$ ", and node $\mathbf{E}$ expresses that "all $P$ 's are not- $Q$ ". The additional requirements demand that these conditions be not 
true together, which enforces that $P$ is not equal to not- $Q$. In set-theoretic terms, the sets $P, Q, \bar{P}, \bar{Q}$ should be non-empty (as in the JK-cube), and not equal.

2.5.6. Gradual extension of the JK cube. Like for the square of opposition and Moretti's cube of opposition, gradual extensions can be proposed for the JK cube. Let us suppose that the front and back of the cube are square of opposition and $\alpha, \epsilon, o, \iota$, and $\alpha^{\prime}, \epsilon^{\prime}, o^{\prime}, \iota^{\prime}$, be the grades associated to vertices $\mathbf{A}, \mathbf{E}, \mathbf{O} \mathbf{I}$, and $\mathbf{a}, \mathbf{e}, \mathbf{o}, \mathbf{i}$. Then, we can define a graded JK cube of opposition in the following way.

Given an involutive negation $n$, a symmetrical conjunction $c$, using the implication associated $i$ and the disjunction, the constraints associated to the cube are given in the following definition (see also Figure 12).

Definition 6. A graded cube of opposition in the sense of Reichenbach is defined by attaching $L$-valued variables $\alpha, \epsilon, o, \iota \alpha^{\prime}, \epsilon^{\prime}, o^{\prime}$ and $\iota^{\prime}$ to each vertex $\mathbf{A}, \mathbf{E}, \mathbf{O}, \mathbf{I}, \mathbf{a}, \mathbf{e} \mathbf{o}, \mathbf{i}$ respectively, in such a way that:

- Front and back facets: $\alpha \iota \epsilon o$ and $\alpha^{\prime} \iota^{\prime} \epsilon^{\prime} o^{\prime}$ are squares of opposition in the sense of Definition 2,

- Side facets: subalterns (entailments):

(o) $i\left(\alpha, \iota^{\prime}\right)=1 ; i\left(\alpha^{\prime}, \iota\right)=1 ; i\left(\epsilon^{\prime}, o\right)=1 ; i\left(\epsilon, o^{\prime}\right)=1$.

- Top and bottom facets:

(p) Contraries: $c\left(\alpha^{\prime}, \epsilon\right)=0 ; c\left(\alpha, \epsilon^{\prime}\right)=0$;

(q) Sub-contraries: $d\left(\iota^{\prime}, o\right)=1 ; d\left(\iota, o^{\prime}\right)=1$.

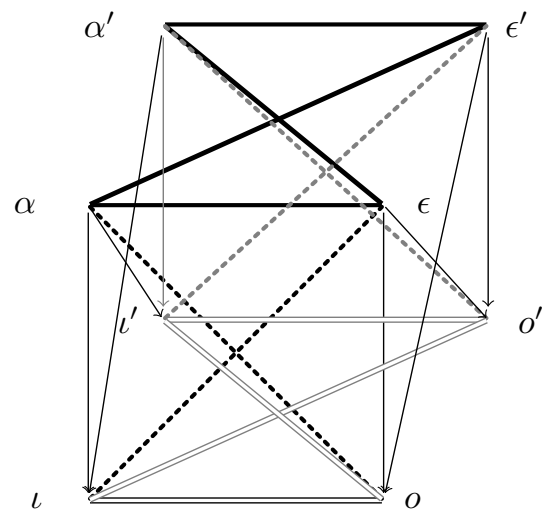

Figure 12. Graded cube of opposition in the sense of Reichenbach

The following counterpart of Proposition 10 can be established [31]:

Proposition 12. Let $\alpha, \iota, o, \epsilon, \alpha^{\prime}, \iota^{\prime}, o^{\prime}, \epsilon^{\prime}$ be eight L-valued variables. Let $n$ be an involutive negation, and $i, c, d$ be many-valued implication, conjunction and disjunction respectively, such that $i$ and $c$ are semi-dual to each other and $d(x, y)=n(c(n(a), n(b))$.

Then $\alpha \iota \epsilon \alpha^{\prime} \iota^{\prime} o^{\prime} \epsilon^{\prime}$ is a cube of opposition in the sense of Reichenbach as soon as we have the following properties: 


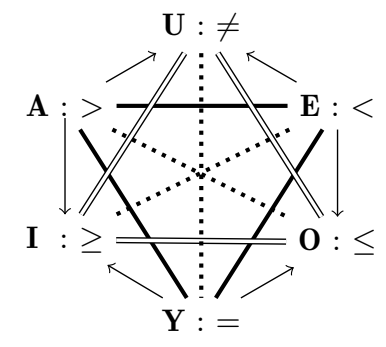

FiguRE 13. Blanché's complete preorder hexagon

- Contradictories: $\alpha=n(o), \epsilon=n(\iota) \alpha^{\prime}=n\left(o^{\prime}\right), \epsilon^{\prime}=n\left(\iota^{\prime}\right)$;

- $i\left(\max \left(\alpha, \alpha^{\prime}\right), \min \left(n(\epsilon), n\left(\epsilon^{\prime}\right)\right)=1\right.$.

The last condition, which is the gradual counterpart of $(\mathbf{A} \vee \mathbf{a}) \rightarrow(\neg \mathbf{E} \wedge \neg \mathbf{e})$, ensures the four subaltern conditions relating the front and back facets of the cube of Figure 12.

The examples of connectives ensuring a graded Reichenbach square are the same as for the graded Moretti cube, since the former differs from the latter by deleting the conditions $c\left(\alpha, \alpha^{\prime}\right)=0$ and $c\left(\epsilon, \epsilon^{\prime}\right)=0$. If we choose a residuated implication and its semi-dual implication, it is enough to use $\alpha, \alpha^{\prime}, \epsilon, \epsilon^{\prime}$ such that $\max \left(\alpha, \alpha^{\prime}\right) \leq \min \left(n(\epsilon), n\left(\epsilon^{\prime}\right)\right)$. It we choose a triangular norm without zero divisors, one must use $\alpha, \alpha^{\prime}, \epsilon, \epsilon^{\prime}$ such that $\max \left(\alpha, \alpha^{\prime}\right)=0$ or $\min \left(n(\epsilon), n\left(\epsilon^{\prime}\right)\right)=1$, that is, if $0<\alpha<1$ or $0<\alpha^{\prime}<1$, we must enforce $\epsilon=\epsilon^{\prime}=0$, and if $0<\epsilon<1$ or $0<\epsilon^{\prime}<1$, we must enforce $\alpha=\alpha^{\prime}=0$.

Gradual versions of the JK cubes of opposition may be found in many settings including possibility theory [19, 31], Shafer's belief function theory [27], qualitative weighted multiple criteria aggregation [27], Sugeno integrals $[27,30]$, and Choquet integrals [30].

\section{Structures of opposition and comparison indices}

This section considers different settings for comparing objects where graded hexagons or cubes of opposition are present. Several results in this section were already proposed in [32].

\subsection{Fuzzy comparison operations and their hexagon of opposition}

Figure 13 represents Blanché's hexagon induced by a complete preorder $\geq$ over a set $U$ based on the tri-partition $A=\left\{(x, y) \in U^{2}: x>y\right\}, E=$ $\left\{(x, y) \in U^{2}: x<y\right\}$ (strict parts of the pre-ordering) and $Y=\left\{(x, x) \in U^{2}\right\}$ (equality). This subsection presents a graded hexagon of opposition for fuzzy comparators on the real line, which extends Blanché's complete preorder hexagon.

It is well-known [57] that a fuzzy set $F$ on a universe $U$ is a mapping $\mu_{F}: U \rightarrow[0,1]$ where $\mu_{F}(x)$ represents the degree of membership of $x$ to 
$F$. We denote by $\operatorname{core}(F)=\left\{x \mid \mu_{F}(x)=1\right\}$ the core of $F$ and $\operatorname{supp}(F)=$ $\left\{x \mid \mu_{F}(u)>0\right\}$ its support. The complement of $F$ is $\bar{F}$ such that $\mu_{\bar{F}}(x)=$ $1-\mu_{F}(x)$. Here, we consider fuzzy intervals on the real line [23].

Consider for simplicity trapezoidal fuzzy intervals. Such a fuzzy set $F$ of the real line has a piecewise linear membership function parameterized by the 4-tuple of reals $(a, b, \alpha, \beta)$ with $\alpha \geq 0, \beta \geq 0$, as pictured on Figure 14, where $\operatorname{core}(F)=[a, b]$ and $\operatorname{supp}(F)=] \bar{a}-\alpha, b+\beta[$.

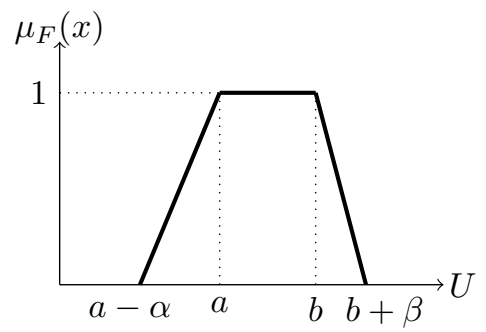

Figure 14. Trapezoidal fuzzy interval

A translation operation consists of adding a constant $c$ to the 4-tuplebased fuzzy set $F=(a, b, \alpha, \beta)$, which yields the trapezoidal fuzzy interval:

$$
F+c=(a+c, b+c, \alpha, \beta) .
$$

The arithmetic negation of $F$ is $-F$ defined by $\mu_{-F}(x)=\mu_{F}(-x)$.

We define three fuzzy relations that express notions of approximately equal to, much greater than, much smaller than as the ones used in the paper [22] for generalizing interval-based temporal reasoning:

- $E$ is a fuzzy approximate equality relation defined by $\mu_{E}(x, y)=\mu_{L}(x-$ $y$ ) where $L$ is a symmetrical fuzzy interval with respect to 0 , namely, $L=(-\delta, \delta, \rho, \rho)$, with $\delta \geq 0, \rho>0$. We have $-L=L$.

- $G$ is a fuzzy relation, representing the concept of much greater than, of the form $\mu_{G}(x, y)=\mu_{K}(x-y)$, where $\mu_{K}$ is an increasing membership function whose support is in the positive real line.

- The fuzzy relation $S$ defined by $\mu_{S}(x, y)=\mu_{-K}(x-y)$ captures the idea of much smaller than. $S$ is the antonym of $G$.

Assume moreover that the three fuzzy sets $-K, L, K$ form a fuzzy partition, namely $\mu_{K}(r)+\mu_{-K}(r)+\mu_{L}(r)=1, \forall r \in \mathbb{R}$, as per Figure 15 for the trapezoidal case.

Using negation $1-(\cdot)$, and Eukasiewicz conjunction one obtains the hexagon of opposition of Figure 16, where:

- The fuzzy version of $\geq$, i.e., approximately greater or equal, is $G \sqcup$ $E$, where $\sqcup$ is defined by Łukasiewicz disjunction, i.e., $\mu_{[G \sqcup E]}(x, y)=$ $\mu_{[K \sqcup L]}(x-y)=\mu_{K}(x-y)+\mu_{L}(x-y)$. It is easy to see that $K \sqcup L=$ $K-2 \delta-\rho=\overline{-K}$ (the overbar denotes fuzzy set complementation: $\left.\mu_{\bar{F}}(x)=1-\mu_{F}(x)\right)$. 


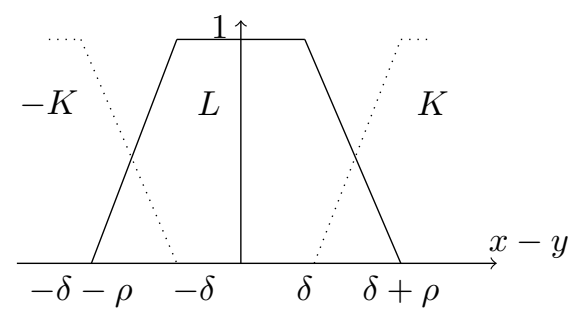

Figure 15. The fuzzy partition of the fuzzy comparison relations

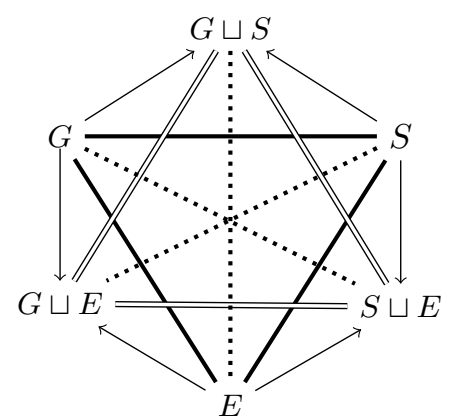

Figure 16. Fuzzy comparator hexagon

- The fuzzy version of $\leq$, i.e., approximately less or equal, is $S \sqcup E$ induced from $(-K) \sqcup L=\bar{K}=(-K)+2 \delta+\rho$.

- The fuzzy version of $\neq$, i.e., not approximately equal, is $G \sqcup S$ induced by $\bar{L}=K \sqcup-K=K \cup-K: \mu_{[G \sqcup S]}(x, y)=1-\mu_{L}(x-y)\left(\right.$ with $\mu_{K \sqcup-K}(x-$ $y)=\mu_{K}(x-y)+\mu_{K}(y-x)=\max \left(\mu_{K}(x-y), \mu_{K}(y-x)\right)=\mu_{K \cup-K}(x-$ $y))$.

It is clear that this is an example of graded hexagon based on Łukasiewicz connectives $\left(n, c_{L}, i_{L}, d_{L}\right)$ where $n(x)=1-x$.

\subsection{Hexagons for similarity and related concepts}

This part presents several hexagons of opposition involving quantitative similarity indices. Let two items be described by their vectors of Boolean features $x=\left(x_{1}, \ldots, x_{q}\right)$ and $y=\left(y_{1}, \ldots, y_{q}\right)$ for a set of attributes $F=$ $\{1, \cdots, i, \cdots, q\}$.

3.2.1. Cardinality-based hexagons. A tripartition of the set of attributes $F$ is formed by the three sets $A g^{+}, A g^{-}$, Dif defined below:

- Positive identity: $\mathrm{Ag}^{+}(x, y)=\left\{i \mid x_{i}=y_{i}=1\right\}$

- Negative identity: $A g^{-}(x, y)=\left\{i \mid x_{i}=y_{i}=0\right\}$

- Opposition: $\operatorname{Dif}(x, y)=\left\{i \mid y_{i} \neq x_{i}\right\}$

This tripartition defines a graded hexagon of opposition based on Lukasiewicz connectives, pictured on Figure 17, an instance of the one on Figure 3, where 


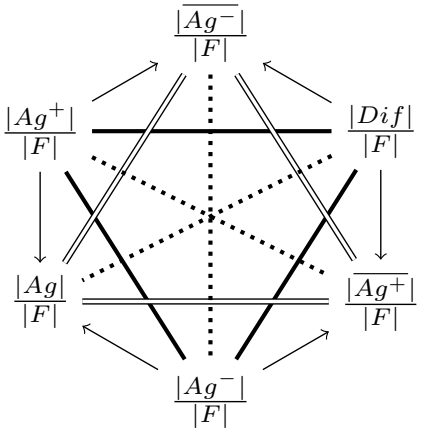

Figure

17

Hexagon of positive and negative identity

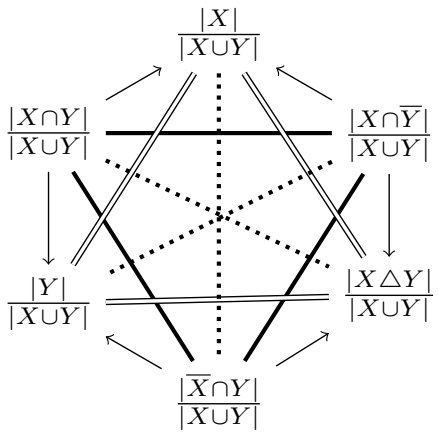

FIGURE

18.

Jaccard index

hexagon

A stands for $A g^{+}(x, y), \mathbf{E}$ for $\operatorname{Dif}(x, y), \mathbf{Y}$ for $A g^{-}(x, y)$, and $A g(x, y)=$ $\left\{i \mid x_{i}=y_{i}\right\}=A g^{+}(x, y) \cup A g^{-}(x, y)$, using relative cardinalities of these sets.

Another similar partition is obtained as follows. Consider $X=\left\{i \mid x_{i}=\right.$ $1\}, Y=\left\{i \mid y_{i}=1\right\}$. A tripartition of $X \cup Y \subset F$ (excluding objects for which $\left.x_{i}=y_{i}=0, \forall i\right)$ can be formed by defining $\mathbf{A}$ as $X \cap Y=A g^{+}(x, y), \mathbf{Y}$ as $\bar{X} \cap Y$ and $\mathbf{E}$ as $X \cap \bar{Y}$. It again yields a graded hexagon of opposition based on Eukasiewicz connectives, pictured on Figure 18, that is again a special case of Figure 3.

We have $\frac{|X \cap Y|}{|X \cup Y|}=1$ if and only if $X=Y$ if and only if $A g(x, y)=F$. Index $\frac{|X \cap Y|}{|X \cup Y|}$ corresponding to vertex $\mathbf{A}$ is clearly Jaccard index, i.e., a wellknown approximate equality measure, while $\frac{|X \triangle Y|}{|X \cup Y|}$ appearing on vertex $\mathbf{O}$ is a difference index (where $X \triangle Y$ is the symmetric difference). However, $\frac{|Y|}{|X \cup Y|}$ is not really a similarity index as it is not symmetrical; $\frac{|X \cap \bar{Y}|}{|X \cup Y|}$ is an opposition index "inside $X$ ", with respect to $Y$. Note also that while $X \cap Y=A g^{+}(x, y)$, $\bar{X} \cap \bar{Y}=\overline{X \cup Y}=A g^{-}(x, y)$. A graded cube involving $\bar{X} \cap \bar{Y}$ together with $X \cap Y, \bar{X} \cap Y$ and $X \cap \bar{Y}$, in terms of cardinalities, is presented in subsection 3.3 .2 .

The hexagons of opposition presented in this paragraph could be generalized, replacing relative cardinalities by weighted averages, or even Choquet integrals following a suggestion in [20].

3.2.2. A binary version of Béziau's analogical hexagon. Béziau's informal analogical hexagon [7] organizes the comparison modalities between items $x$ and $y$ described as above by their respective Boolean attribute values $x_{i}$ and $y_{i}$. Six comparison modalities between $x$ and $y$ can be defined in this framework and can be the vertices of a hexagon represented in Figure 19. 
Namely the following pairs of contradictories, expressed in the terminology of the previous subsection:

- Opposition and Similarity, respectively $A g(x, y)=\emptyset$ and $A g(x, y) \neq \emptyset$

- Identity and Difference, respectively $A g(x, y)=F$ and $A g(x, y) \neq F$

- Analogy and Non-Analogy, respectively $\emptyset \neq A g(x, y) \neq F$, and $A g(x, y)=$ $F$ or $A g(x, y)=\emptyset$

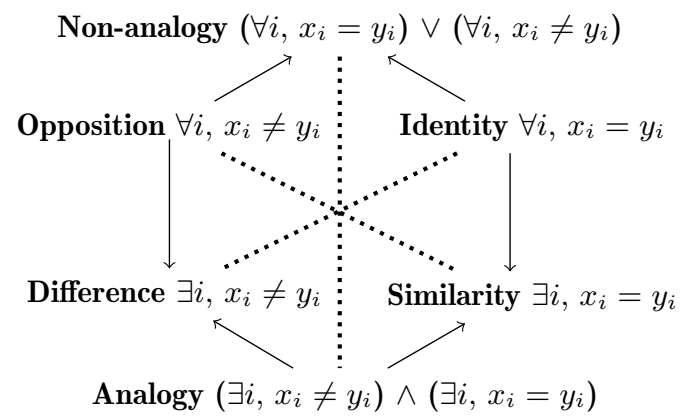

Figure 19. A binary version of Béziau's analogical hexagon

Note that Analogy, viewed here as a conjunction between Difference and Similarity (expressed in a weak form), is both a matter of dissimilarity and resemblance, an idea already present in the logical rendering of an analogical proportion proposed in [48], although modeled differently.

3.2.3. A graded version of Béziau's analogical hexagon. Suppose from now on that item attributes map to a totally ordered value scale $L$ with least and greatest elements respectively denoted by 0 and 1 . The scale $L$ is supposed to be equipped with an involutive negation $n$, such as $1-(\cdot)$, i.e., order-reversing on $L$. On each attribute, equality and difference are evaluated by means of similarity measures $\mu_{S_{i}}: L \times L \rightarrow L$ and dissimilarity measures $\mu_{D_{i}}: L \times L \rightarrow L$. It is natural to assume that $\mu_{S_{i}}=n\left(\mu_{D_{i}}\right)$ for an involutive negation $n$. The vector of similarities between $x$ and $y$ is $\mu_{S}(x, y)=$ $\left(\mu_{S_{1}}\left(x_{1}, y_{1}\right), \cdots, \mu_{S_{q}}\left(x_{q}, y_{q}\right)\right)$, while it is $\mu_{D}(x, y)=\left(\mu_{D_{1}}\left(x_{1}, y_{1}\right), \cdots, \mu_{D_{q}}\left(x_{q}, y_{q}\right)\right)$ for dissimilarity. For any two items $x$ and $y$, it is also supposed that separability holds, namely: $\mu_{S_{i}}\left(x_{i}, y_{i}\right)=1$ (resp. $\mu_{D_{i}}\left(x_{i}, y_{i}\right)=1$ ) if and only if $x_{i}$ and $y_{i}$ are perfectly similar: $x_{i}=y_{i}$.

If we consider Lukasiewicz triplet $\left(i_{L}, c_{L}, d_{L}\right)$ then a hexagon of opposition is obtained since $\min _{i} \mu_{D_{i}}\left(x_{i}, y_{i}\right) \leq \max _{i} \mu_{D_{i}}\left(x_{i}, y_{i}\right)$ (see Figure 20), in agreement with Proposition 4.

In this case, two items are perfectly analogical if they are perfectly similar and perfectly different. They are non-analogical if $\min _{i} \mu_{D_{i}}\left(x_{i}, y_{i}\right)+$ $\min _{i} \mu_{S_{i}}\left(x_{i}, y_{i}\right) \geq 1$, i.e., $\min _{i} \mu_{D_{i}}\left(x_{i}, y_{i}\right) \geq \max _{i} \mu_{D_{i}}\left(x_{i}, y_{i}\right)$. In this case, similarity and dissimilarity measures are constant and equal to 1 or 0 . Then, the two items are either perfectly identical or perfectly opposite on a given attribute. 


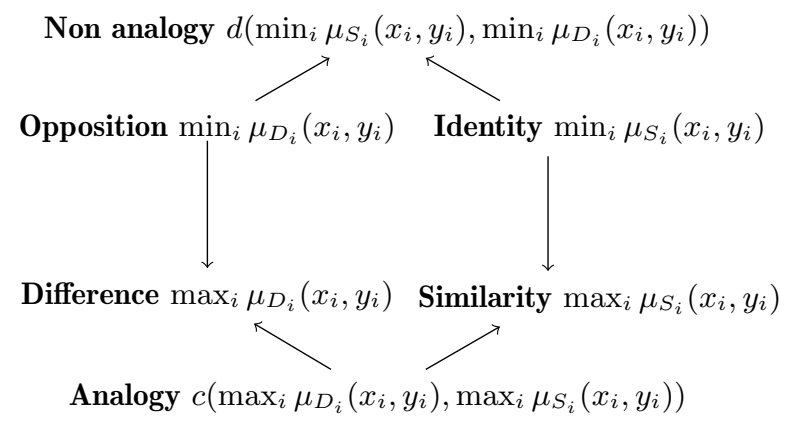

FiguRE 20. Graded analogical hexagon

3.2.4. Weighted attributes in the graded analogical hexagon. Borrowing from possibility theory for fuzzy events $[58,31]$, the previous hexagon can be extended to gradual modalities using weighted min and max operators on vertices. Operations min and max are qualitative elementary operators that can be extended by means of importance weights or priorities $\pi_{i}$ assigned to attributes. The closer $\pi_{i}$ to 1 , the more important the attribute. Such importance weights may alter local evaluations in various ways [29], leading to operators of the form (here applied to a vector $t$ )

$$
M I N_{\pi}^{\rightarrow}(t)=\min _{i=1}^{q} \pi_{i} \rightarrow t_{i}, \quad M A X_{\pi}^{\otimes}(t)=\max _{i=1}^{q} \pi_{i} \otimes t_{i},
$$

where $(\rightarrow, \otimes)$ is a pair of semi-dual implication and conjunction.

We build a hexagon presented in Figure 21 where we use shorthand $I d$., Op., Dif., Sim., An. and NonAn. for the vertices of the analogical hexagon. Under mild conditions already pointed out for possibility and necessity of

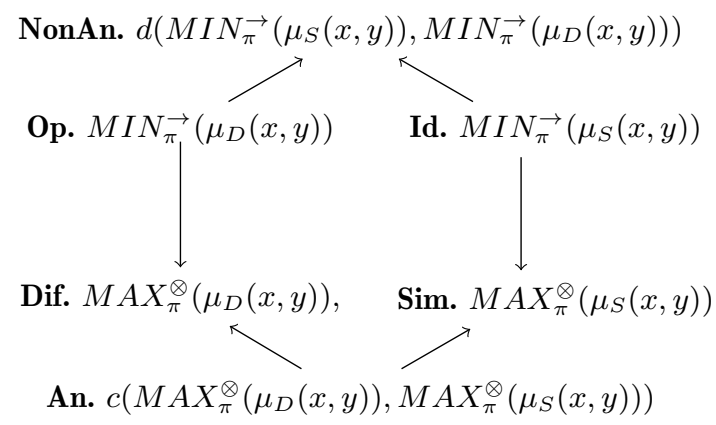

Figure 21. Fuzzy weighted analogical hexagon

fuzzy events ([31], Proposition 17), we can build a hexagon of opposition based on Łukasiewicz connectives: 
Proposition 13. If $n$ is an involutive negation, and $c_{L}$ is the Eukasiewicz triangular norm, then the fuzzy weighted analogical hexagon of Figure 21, obtained from the Eukasiewicz triplet $\left(i_{L}, c_{L}, d_{L}\right)$, is a hexagon of opposition as soon as there is an attribute such that $\pi_{i}=1$, and implication $\rightarrow$ is such that $n(a) \rightarrow 0 \leq n(a \rightarrow 0)$.

Proof Let $j$ be an index such that $\pi_{j}=1$. Then $\min _{i=1}^{q} \pi_{i} \rightarrow t_{i} \leq$ $1 \rightarrow t_{j} \leq 1 \otimes t_{j} \leq \max _{i=1}^{q} \pi_{i} \otimes t_{i}$ provided that $1 \rightarrow t_{j} \leq n\left(1 \rightarrow n\left(t_{j}\right)\right)$, which is the same as the assumption $n\left(1 \rightarrow t_{j}\right) \geq 1 \rightarrow n\left(t_{j}\right)$. So we get $\operatorname{MIN}_{\pi}^{\rightarrow}\left(\mu_{D}(x, y)\right) \leq M A X_{\pi}^{\otimes} \mu_{D}(x, y)$, that is $\alpha \leq \iota$ in the hexagon. The rest follows by Proposition 4

We give three examples of semi-dual pairs $(\rightarrow, \otimes)$ where this proposition applies:

1. Kleene-Dienes. $M I N_{\pi}^{\rightarrow_{K D}}(t)=\min _{i=1}^{q} \max \left(1-\pi_{i}, t_{i}\right) \leq M A X_{\pi}^{\otimes_{K D}}(t)=$ $\max _{i=1}^{q} \min \left(\pi_{i}, t_{i}\right)$ is well-known: they are the standard versions of the weighted min and weighted max operations [24]. Then two items will be perfectly opposite (resp. identical) if for each attribute either its importance is zero, or dissimilarity (resp. similarity) between items is perfect. They will be perfectly different (resp. similar) if there exists at least one attribute with importance 1 for which there is perfect dissimilarity (resp. similarity) between items.

2. Gödel implication: Since $(1-a) \rightarrow_{G} 0=0$, it follows that $M I N_{\pi} \rightarrow^{G}\left(\mu_{D}(x, y)\right) \leq$ $M A X_{\pi}^{\otimes_{G}} \mu_{D}(x, y)$ if $\pi_{i}=1$ for some $i$. The weights $\pi_{i}$ only play the role of thresholds. Then two items will be perfectly opposite (resp. identical) if all local dissimilarities (resp. similarities) are above their thresholds. They will be perfectly different (resp. similar) if there exists at least one attribute with non-zero importance $\pi_{i}$ and perfect dissimilarity (resp. similarity) between items.

3. Contrapositive Gödel implication: We can check that $(1-a) \rightarrow_{G C}$ $0=1-a=1-\left(a \rightarrow_{G C} 0\right)$. It follows that $\operatorname{MIN}_{\pi} \rightarrow_{G C}\left(\mu_{D}(x, y)\right) \leq$ $M A X_{\pi}^{\otimes_{G C}} \mu_{D}(x, y)$, i.e., $\min _{i \mid \pi_{i}>\mu_{D_{i}}\left(x_{i}, y_{i}\right)} 1-\pi_{i} \leq \max _{i \mid \mu_{D_{i}}\left(x_{i}, y_{i}\right)>1-\pi_{i}} \pi_{i}$. Then two items will be perfectly opposite (resp. identical) if all local dissimilarities (resp. similarities) are above their thresholds. They will be different (resp. similar) if there exists at least one attribute with $\pi_{i}=1$ and non-zero dissimilarity (resp. similarity) between items.

A further extension of the analogical hexagon involving statements with weakened quantifiers, such as " $x$ and $y$ are (highly) similar on at least $k$ over $q$ attributes" has been outlined and discussed in [32]. Since the treatment of such quantifiers is a topic in itself, and would be relevant as well for the other structures of opposition (square, cubes), we leave it aside in this paper.

\subsection{Cubes of opposition in the comparison of items}

It seems that cubes of opposition, in spite of their noticeable applications in various settings as already mentioned in the previous section, do not bring 
much to the discussion of the various points of view for comparing items. However, some comments are worth making for each of the two cubes studied in this paper.

We use the notations of subsection 3.2. We consider two items $x$ and $y$ described by vectors of Boolean features, namely $x=\left(x_{1}, \ldots, x_{q}\right)$ and $y=\left(y_{1}, \ldots, y_{q}\right)$, for a set of attributes $F=\{1, \cdots, i, \cdots, q\}$.

3.3.1. The JK cube in the comparison of items. The hexagons of subsections $3.2 .2,3.2 .3$ and 3.2 .4 , or more precisely the square part corresponding to Identity, Opposition, Difference and Similarity can be extended in a JK cube of opposition, as presented in Figure 22. The eight different evaluations are based on the vector of similarities and the vector of dissimilarities between $x$ and $y: \mu_{S}(x, y)$ and $\mu_{D}(x, y)=1-\mu_{S}(x, y)$, and $\pi_{i} \in L$ denotes the level of importance of attribute $i$ (the larger $\pi_{i}$ the more important $i$ ). A double normalization is assumed $\exists i, \pi_{i}=1$ and $\exists j, \pi_{j}=0$. This cube is nothing be the one of possibility theory for fuzzy events $[28,31]$, where the fuzzy events pertain here to the similarity / dissimilarity of items, and where the possibility distribution refers to the importance of the attributes. The front facet of the cube is the original square present in the hexagons of the abovementioned subsections.

As can be seen, the back facet appears to be a copy of the front facet where the weights of importance are reversed. Let us consider the vertex a to see what kind of new evaluations is brought by the cube. Let us assume that the implication, like, e.g., Łukasiewicz' implication, is equal to its contrapositive, namely $a \rightarrow b=n(b) \rightarrow n(a)$ with $n(a)=1-a$. Then the expression in vertex a evaluates to what extent all the attributes on which $x$ and $y$ are (highly) similar are important (since $\mu_{S_{i}}\left(x_{i}, y_{i}\right)=1-\mu_{D_{i}}\left(x_{i}, y_{i}\right)$ ), while the expression in vertex $\mathbf{A}$ evaluates to what extent all important attributes are attributes on which $x$ and $y$ are (highly) similar. The former expression (vertex a) leaves room to the possibility of not being similar on some important attributes, but when similarity takes place it is only on important attributes, while the latter expression (vertex $\mathbf{A}$ ) allows to be similar also on some non important attributes.

3.3.2. Moretti's cube and comparisons. We have already mentioned two examples of quadri-partitions giving birth to a cube of opposition in the sense of Moretti. The first one pertains to binary preference attitudes ( $x$ preferred to $y, y$ preferred to $x$, indifference between $x$ and $y, x$ and $y$ not comparable). This can be extended to gradual relations [35] in agreement with the gradual extension of Moretti's cube. The disjunctions of three of the four relations are not always easy to understand (even if some are: $x$ preferred to $y$ or $y$ preferred to $x$ or $x$ and $y$ are indifferent clearly means $x$ and $y$ are comparable); they can however be understood as negations of the 4 original relations.

The second example of Moretti's cube for comparison indices relies on conjunctions of pairs of propositions that are negated or not and proportions of objects satisfying these propositions. This is an example of the graded 


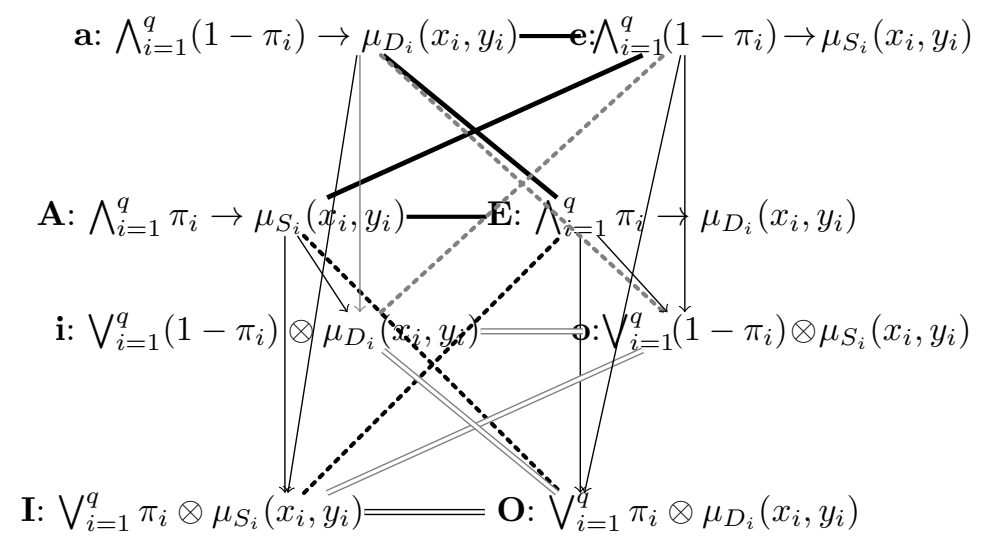

Figure 22. The JK cube for comparison

cube of Figure 8. Recall $X=\left\{i \mid x_{i}=1\right\}, Y=\left\{i \mid y_{i}=1\right\}$ and their complements $\bar{X}=\left\{i \mid x_{i}=0\right\}, \bar{Y}=\left\{i \mid y_{i}=0\right\}$. We assume there are no objects for which $x_{i}=0, \forall i, x_{i}=1, \forall i$, etc). So $X \neq \emptyset, \bar{X} \neq \emptyset, Y \neq \emptyset$, $\bar{Y} \neq \emptyset$. Moreover $X \neq Y$ must be assumed. Clearly, we have $F=U=$ $(X \cap Y) \cup(\bar{X} \cap Y) \cup(X \cap \bar{Y}) \cup(\bar{X} \cap \bar{Y})$. Thus $X \cap Y, \bar{X} \cap Y, X \cap \bar{Y}, \bar{X} \cap \bar{Y}$ form a quadri-partition of $F$ (if none of them are empty and $X \neq Y$ ), and we obtain the cube of Figure 8, which is a gradual counterpart of the one of Figure 6. This completes the hexagon of Figure 17 which only involves $X \cap Y$, $\bar{X} \cap Y$, and $X \cap \bar{Y}$, but not $\bar{X} \cap \bar{Y}$.

\section{Conclusion}

In the first part of this paper, we have systematically studied the logical constraints underlying important structures of opposition, namely the traditional square, Blanché's hexagon, Johnson and Keynes octagon and the related cube proposed by Dubois and Prade, Reichenbach's cube and Moretti's cube. We have also provided their gradual extensions in order to accommodate the situation when the satisfaction of statements associated to the vertices is a matter of degree. In the second part, we have illustrated these structures on statements pertaining to the comparison of items. As in other cases, these structures of opposition organize the relationships between various modalities and can survive under various gradual extensions thereof. Besides, the compatibility of weakened universal quantifiers ("most" instead of "all") and strengthened existential quantifiers ("a few" instead of "at least one") with these structures is a matter of further investigation.

\section{Acknowledgements}

The second author thanks Lorenz Demey (Center for Logic and Philosophy of Science, Leuven, Belgium) for interesting discussions on cubes of opposition in Kolymbari (Crete) in early November 2018 at the 6th world congress on the Square of Opposition. The authors are indebted towards an anonymous 
reviewer for pointing out references to Johnson-Keynes octagon as the proper ancestor of their cube.

\section{References}

[1] L. Amgoud, H. Prade: A formal concept view of abstract argumentation. Proc. Europ. Conf. on Symbolic and Quantitative Approaches to Reasoning with Uncertainty (ECSQARU'13), (L. C. van der Gaag, ed.), Utrecht, July 8-10, Springer, LNCS, vol. 7952, 1-12, 2013.

[2] Anonymous. Syllogisme. In Encyclopédie ou Dictionnaire Raisonné des Sciences des Arts et Métiers, de D. Diderot and J. d'Alembert. 1751-1772.

[3] C. Alsina: On a family of connectives for fuzzy sets. Fuzzy Sets and Systems. vol. 16, (3), pp. 231-235 (1985).

[4] N. Barbot, L. Miclet, H. Prade: The analogical cube of opposition. In Handbook of abstracts of the 6th World Congress on the Square of Opposition, (J. Y. Béziau, A. Buchsbaum, I. Vandoulakis, eds.), Crete, Nov. 1-5, pages 9-10. 2018.

[5] N. Barbot, L. Miclet, H. Prade: A new perspective on analogical proportions. Proc. 15th Europ. Conf. on Symbolic and Quantitative Approaches to Reasoning with Uncertainty (ECSQARU'19), (G. Kern-Isberner, Z. Ognjanović, eds.), Belgrade, Sept. 18-20, Springer, LNCS, to appear, 2019.

[6] J. Y. Béziau: New light on the square of oppositions and its nameless corner. Logical Investigations, 10, 218-233, 2003.

[7] J.-Y. Béziau: The power of the hexagon, Logica Universalis, 6 (1-2) : 1-43, 2012.

[8] J.-Y. Béziau: There is no cube of opposition. In Beziau, J.-Y., Basti, G., eds, The Square of Oppositions: A Cornerstone of Thought, Studies in Universal Logic, Birkaüser Verlag 179-193, 2017.

[9] J.-Y. Béziau: An analogical hexagon. Int. J. Approx. Reasoning, 94, 1-17, 2018.

[10] J. Y. Béziau, G. Payette (eds.): The Square of Opposition. A General Framework for Cognition. Peter Lang, 2012.

[11] J. Y. Béziau, K. Gan-Krzywoszyńska (eds.): Handbook of abstracts of the 2nd World Congress on the Square of Opposition, Corte, Corsica, June 17-20, 2010.

[12] J. Y. Béziau, K. Gan-Krzywoszyńska (eds.): Handbook of abstracts of the 3rd World Congress on the Square of Opposition, Beirut, Lebanon, June 26-30, 2012.

[13] J. Y. Béziau, K. Gan-Krzywoszyńska (eds.): Handbook of abstracts of the 4th World Congress on the Square of Opposition, Roma, Vatican, May 5-9, 2014.

[14] J. Y. Béziau, A. Buchsbaum, M. Correia (eds.): Handbook of abstracts of the 5th World Congress on the Square of Opposition, Easter Island, Nov. 11-15, 2016.

[15] R. Blanché: Structures Intellectuelles. Essai sur l'Organisation Systématique des Concepts. Librairie philosophique J. Vrin, Paris, 1966.

[16] R. Blanché: Sur l'opposition des concepts, Theoria, 19 : 89-130, 1953.

[17] Ch. de Bovelles: L'Art des Opposés. Texte et traduction par P. Magnard précédé d'un essai, Soleil Noir, avec 37 reproductions. Librairie Philosophique J. Vrin, Paris, 1984.

[18] D. Ciucci, D. Dubois, H. Prade: Structures of opposition in fuzzy rough sets. Fundam. Inform. 142, 1-19, 2015. 
[19] D. Ciucci, D. Dubois, H. Prade: Structures of opposition induced by relations. The Boolean and the gradual cases. Annals of Mathematics and Artificial Intelligence, 76 (3), 351-373, 2016.

[20] G. Coletti, D. Petturiti, B. Vantaggi: Fuzzy weighted attribute combinations based on similarity measures. Proc. 14th Europ. Conf. on Symbolic and Quantitative Approaches to Reasoning with Uncertainty (ECSQARU'17), (A. Antonucci, L. Cholvy, O. Papini, eds.), Lugano, July 10-14, Springer, LNCS 10369, 364-374, 2017.

[21] D. Dubois: Generalized probabilistic independence and its implications for utility. Operations Research Letters, 5(5), 255-260, 1986.

[22] D. Dubois, A. HadjAli, H. Prade: Fuzziness and Uncertainty in Temporal Reasoning. J. of Universal Computer Science 9 : 1168-1194, 2003.

[23] D. Dubois, E. Kerre, R. Mesiar, H. Prade: Fuzzy interval analysis. In: Fundamentals of Fuzzy Sets, Dubois,D. Prade,H., Eds: Kluwer, Boston, Mass, The Handbooks of Fuzzy Sets Series, 483-581, 2000.

[24] D. Dubois, H. Prade: Weighted minimum and maximum operations in fuzzy set theory. Information Sciences, 39 (2): 205-210, 1986.

[25] D. Dubois, H. Prade: From Blanché's hexagonal organization of concepts to formal concept analysis and possibility theory. Logica Univers., 6, 149-169, 2012.

[26] D. Dubois, H. Prade: Possibility Theory - An Approach to Computerized Processing of Uncertainty, Plenum Press, 1988.

[27] D. Dubois, H. Prade: Gradual structures of oppositions. In: Esteva, F., Magdalena, L., Verdegay, J.L. (eds.) Enric Trillas: Passion for Fuzzy Sets, Studies in Fuzziness and Soft Computing, vol. 322, 79-91. Springer, 2015.

[28] D. Dubois, H. Prade, A. Rico: The cube of opposition: A structure underlying many knowledge representation formalisms. Proc. 24th Int. Joint Conf. on Artificial Intelligence (IJCAI'15), Buenos Aires, July 25-31 (Q. Yang, M. Wooldridge, eds.), AAAI Press, 2015, 2933-2939.

[29] D. Dubois, H. Prade, A. Rico: Residuated variants of Sugeno integrals: Towards new weighting schemes for qualitative aggregation methods. Information Sciences : 94-105, 2016.

[30] D. Dubois, H. Prade, A. Rico: Organizing families of aggregation operators into a cube of opposition. In: Granular, Soft and Fuzzy Approaches for Intelligent Systems, Dedicated to Professor Ronald R. Yager, (Kacprzyk, J., Filev, D., Beliakov, G., eds.), Studies in Fuzziness and Soft Computing Volume 344, 2745, Springer Verlag, 2017.

[31] D. Dubois, H. Prade, A. Rico: Graded cubes of opposition and possibility theory with fuzzy events. Int. J. of Approximate Reasoning, 84, 168-185, 2017.

[32] D. Dubois, H. Prade, A. Rico: Fuzzy extensions of conceptual structures of comparison. Proc. 17th Int. Conf. on Information Processing and Management of Uncertainty in Knowledge-Based Systems (IPMU'18), Cádiz, June 11-15, Part I, (J. Medina, M. Ojeda-Aciego, J. L. Verdegay Galdeano, D. A. Pelta, I. P. Cabrera, B. Bouchon-Meunier, R. R. Yager (eds.), Communications in Computer and Information Science, vol. 853, Springer, 710-722, 2018.

[33] S. Dupleix: De l'opposition des énonciations. Book IV, chap. 10, in La logique ou Art de Discourir et de Raisonner. 1607. Reprinted by Fayard, Paris, 1984. 
[34] J. C. Fodor: Contrapositive symmetry of fuzzy implications. Fuzzy Sets and Systems 69, 141-156 (1995).

[35] J. C. Fodor, M. Roubens: Fuzzy Preference Modelling and Multicriteria Decision Support. Kluwer Acad. Publ., Dordrecht, 1994.

[36] M. J. Frank: On the simultaneous associativity of $F(x, y)$ and $x+y-F(x, y)$. Aequationes Math., 19, 194-226, 1979.

[37] P. Jacoby. A triangle of opposites for types of propositions in Aristotelian logic. The New Scholasticism, XXIV (1), 32-56, 1950.

[38] W.E. Johnson. Logic. Part I, Ch. 9. Cambridge University Press, p. 142, 1921.

[39] J. N. Keynes. Studies and Exercises in Formal Logic, Part II, Ch. IV (Third Edition). MacMillan, p. 144, 1894.

[40] E.P. Klement, R. Mesiar, E. Pap: Triangular Norms, Kluwer Academic, Dordrecht, 2000.

[41] D. Luzeaux, J. Sallantin, C. Dartnell: Logical extensions of Aristotle's square. Logica Universalis, 2, 1, 167-187, 2008.

[42] A. Moretti: Geometry for modalities? Yes: Through N-opposition theory? In: Aspects of Universal Logic, (Béziau J.-Y., Costa Leite A., and Facchini A. eds.), University of Neuchâtel, Neuchâtel, 102-145, 2004.

[43] A. Moretti: The geometry of opposition. Phd Thesis. University of Neuchâtel, 2009.

[44] A. Moretti: The geometry of standard deontic logic. Logica Universalis, 3, 1, 19-57, 2009.

[45] T. Parsons: The traditional square of opposition. In: E. N. Zalta, editor, The Stanford Encyclopedia of Philosophy, 2008.

[46] R. Pellissier: "Setting" $n$-opposition. Logica Universalis 2(2), 235-263 (2008)

[47] R. Pellissier: 2-opposition and the topological hexagon. In: New Perspectives on the Square of Opposition, (J.-Y. Béziau, G. Payette, eds.), Peter Lang, Bern, 2012 .

[48] H. Prade, G. Richard: From analogical proportion to logical proportions. Logica Universalis, 7, 441-505, 2013.

[49] H. Reichenbach: The syllogism revised. Philosophy of Science,19 (1), 1-16, 1952.

[50] M. Roubens, Ph. Vincke: Preference Modeling. LNEMS 250, Springer Verlag, Berlin, 1985.

[51] E. H. Ruspini: A new approach to clustering. Information and Control, 15, 22-32, 1969.

[52] P. Sauriol: Remarques sur le théorie de l'hexagone logique de Blanché. Canadian Philosophical Review, 7 (3), 374-390, 1968.

[53] A. Sesmat: Logique II: Les Raisonnements, la Logistique. Hermann, Paris. 1951.

[54] W. of Sherwood: William of Sherwood's Introduction to Logic. University of Minnesota Press, Minneapolis, with translation, introduction and notes by N. Kretzmann, 1966.

[55] H. Smessaert: On the 3D visualisation of logical relations. Logica Universalis, 3, 303-332, 2009.

[56] B. Van de Walle, B. De Baets, E. E. Kerre: Characterizable fuzzy preference structures. Annals OR 80: 105-136 (1998) 
[57] L.A. Zadeh: Fuzzy sets. Information and Control, 8:338-353, 196

[58] L. A. Zadeh: Fuzzy sets as a basis for a theory of possibility. Fuzzy Sets and Systems 1 (1978) 3-28.

Didier Dubois, Henri Prade

Institut de Recherche en Informatique de Toulouse (IRIT)

Université Paul Sabatier, 118 route de Narbonne

31062 Toulouse Cedex 9, France

e-mail: $\{$ dubois, prade\}@irit.fr

Agnès Rico

Equipe de Recherche en Ingénierie des Connaissances - ERIC

Université Claude Bernard Lyon 1, Lyon, France

e-mail: agnes.rico@univ-lyon1.fr 